\title{
Relations and trails in lattices of projections in $\mathrm{W}^{*}$-algebras
}

\author{
Edward Kissin
}

May 28, 2019

\begin{abstract}
The paper studies HH-relations in the lattices $P(M)$ of all projections of $\mathrm{W}^{*}$-algebras $M$. If $M$ is a finite algebra, all these relations are generated by trailes in $P(M)$. If $M$ is an infinite countably decomposable factor, they are either generated by trails, or associated with them.
\end{abstract}

\section{Introduction and preliminaries}

The aim of this paper is to describe $\mathbf{H H}$-relations in the lattices of all projections of $\mathrm{W}^{*}$-algebras.

A partially ordered set $(Q, \leq)$ with a reflexive, anti-symmetric, transitive relation $\leq$ is a lattice if all $a, b \in Q$ have a least upper bound $a \vee b$ and a greatest lower bound $a \wedge b$. It is complete if each subset $G \subseteq Q$ has a least upper bound $\vee G$ and a greatest lower bound $\wedge G$.

Let $\ll$ be a relation in $(Q, \leq)$ (we only consider reflexive relations $\ll$ in $Q$ stronger than $\leq$, i.e., $a \ll b$ implies $a \leq b$ for $a, b \in Q)$. For $a<b$ in $Q$, set

$$
[a, \ll]=\{x \in Q: a \ll x\},[\ll, b]=\{x \in Q: x \ll b\} .
$$

The relation $\ll$ is up-expanded if $[a, \ll]$ has a least upper bound for each $a \in Q$; it is down-expanded if $[\ll, b]$ has a greatest lower bound for each $b \in Q$. We write $\mathbf{0}=\wedge Q$ and $\mathbf{1}=\vee Q$.

Amitsur [A1] (see also [Gr]) defined $\mathbf{H}$ - and dual $\mathbf{H}$-relations on complete lattices as follows:

$$
\begin{aligned}
& \ll \text { is an H-relation if } a \ll b \text { and } a \leq c \text { imply } c \ll b \vee c, \\
& \ll \text { is a dual } \mathbf{H} \text {-relation if } a \ll b \text { and } c \leq b \text { imply } a \wedge c \ll c \text {. }
\end{aligned}
$$

for $a, b, c \in(Q, \leq)$. An $\mathbf{H}$-relation is an $\mathbf{R}$-order if it is transitive and up-expanded; a dual $\mathbf{H}$ relation is a dual $\mathbf{R}$-order if it is transitive and down-expanded. We say that $\ll$ is

an $\mathbf{H H}$-relation if it is an $\mathbf{H}$ - and a dual $\mathbf{H}$-relation;

an $\mathbf{R R}$-order if it is an $\mathbf{R}$ - and a dual $\mathbf{R}$-order.

Amitsur [A1] developed the theory of radicals in lattices and proved that if $\ll$ is an $\mathbf{R}$-order (resp. a dual R-order), then $Q$ has a unique «-radical (resp. a dual «-radical). In [A2] he applied this to the study of the theory of modules and rings. The problem naturally arises to give an intrinsic description of HH-relations and RR-orders in various lattices. This will allow to describe the radicals they generate and, using the fact that these radicals are invariant for all automorpisms of lattices, to investigate their properties. 
Let $\mathfrak{S}_{A}$ be the set of all Lie subalgebras of finite codimension in a Banach Lie algebra $A$. Using the relation $\ll_{\infty}$ in the lattice $\operatorname{Id}_{A}^{\text {char }}$ of all characteristic Lie ideals of $A$ (invariant for all derivations of $A$ ), one obtains that the condition $\cap\left\{S: S \in \mathfrak{S}_{A}\right\}=\{0\}$ implies that the dual $\ll_{\infty}^{\triangleleft}$ radical $\rho=\{0\}$ and there is a descending series $\left(I_{\lambda}\right)_{1 \leq \lambda \leq \gamma}$ of characteristic Lie ideals of $A$ such that $\operatorname{dim}\left(I_{\lambda} / I_{\lambda+1}\right)<\infty$ for $\lambda \neq \gamma, I_{1}=A$ and $I_{\gamma}=\{0\}$.

In $[\mathrm{KST}]$ the author, Shulman and Turovskii studied HH-relations in the lattices $\operatorname{Id}_{\mathcal{A}}$ of all ideals of $\mathrm{C}^{*}$-algebras $\mathcal{A}$. Although it was not possible to describe all of them, it was shown that many well known relations in $\operatorname{Id}_{\mathcal{A}}$ are $\mathbf{H H}$-relations. This allowed to establish that many important results for $\mathrm{C}^{*}$-algebras follow from the theory of $\mathbf{H H}$-relations in abstract lattices. For example, the largest GCR-ideal in any $\mathrm{C}^{*}$-algebra $\mathcal{A}$ is the radical generated by some $\mathbf{H H}$-relation in $\operatorname{Id}_{\mathcal{A}}$.

In this paper we study $\mathbf{H H}$-relations in the complete lattices $Q=P(M)$ of all projections in $\mathrm{W}^{*}$-algebras $M$. These lattices are widely investigated, but not much is known about various types of relations in them. In particular, the structure of $\mathbf{H}$ - and dual $\mathbf{H}$-relations in $Q$ and the nature of the radicals generated by them is very complicated and difficult to investigate. The situation becomes tractable, if we consider $\mathbf{H H}$-relations in $Q$. It turns out that these relations are closely linked to trails in the lattice $Q$ and to the traces on $M$. This allows us to describe a large variety of HH-relations in $P(M)$ and to give a full description of these relations in the case when $M$ are countably decomposable factors.

We call a subset $K$ in $Q$ a trail (cf. Exercises V.1.5 (c) [T]) if,

$$
q \precsim p \in K \text { implies } q \in K(\precsim \text { is the Murray-vonNeumann relation in } Q \text { ). }
$$

Each trail $K$ generates an HH-relation $\ll_{K}$ on $Q: p \ll_{K} q$ if $q-p \in K$. In Corollary 4.8 we prove that in finite $\mathrm{W}^{*}$-algebras the converse is also true: each $\mathbf{H H}$-relation is generated by a trail. Moreover, in type $\mathrm{I}_{n}$ factors each trail corresponds to the value of the trace $\tau$ on $Q$; in type $\mathrm{II}_{1}$ factors two trails correspond to the same value of $\tau$ (Theorem 7.2).

For infinite $\mathrm{W}^{*}$-algebras, the structure of $\mathbf{H H}$-relations is more complicated. Not all of them are generated by trails. For each trail $K$ in $Q$, there is also an HH-relation $\ll_{\perp}^{K}$ associated with $K$, but not generated by any trail (Theorem 5.3). For example, in type $\mathrm{I}_{\infty}$ factor, for each $n \in \mathbb{N}$, the relation $p \ll_{\perp}^{K_{\omega_{n}}} q$ if $\operatorname{codim}(q-p)>k$, is an $\mathbf{H H}$-relation. Apart from $\mathbf{H H}$-relations generated by trails and associated with trails, $Q$ may have many "mixed type" HH-relations. However, if $M$ is a countably decomposable type $\mathrm{I}_{\infty}$, or $\mathrm{II}_{\infty}$ factor, then all $\mathbf{H H}$-relations in $Q$ are either generated by, or associated with trails. In type $\mathrm{I}_{\infty}$ factors each trail corresponds to the value of the trace $\tau$ on $Q$, so that two $\mathbf{H H}$-relations correspond to this value. In type $\mathrm{II}_{\infty}$ factors two trails correspond to the same value of $\tau$, so that four $\mathbf{H H}$-relations correspond to this value (Theorem 7.8).

Countably decomposable type III factors do not have faithful tracial weights and their lattices only have two trails $K_{\mathbf{0}}=\{\mathbf{0}\}$ and $K_{\mathbf{1}}=Q$. It turns out that their sets of $\mathbf{H H}$-relations are also very simple - they only have three $\mathbf{H H}$-relations. Two are trivial: $=$ and $\leq$, they are generated by trails. The third relation $\ll_{\perp}^{K_{0}}$ is associated with $K_{0}$ and almost coincides with $\leq$ (Theorem 7.9).

The link between the transitive $\mathbf{H H}$-relations and the trails in $Q=P(M)$ for all $\mathrm{W}^{*}$-algebras $M$, becomes very strong and does not depend on the type of $M$ : A relation $\ll$ is an $\mathbf{H H}$-order if and only if it is generated by a trail $K$ in $Q\left(\ll=\ll_{K}\right)$ and $K$ is also a sublattice (Theorem 6.1).

Finally, RR-orders constitute a set isomorphic to the set of all central projections $z$ in $Q$ : A relation $\ll$ is an $\mathbf{R R}$-order if and only if it is generated by a trail $Q z$, i.e., $\ll=\ll_{Q z}$ (Corollary 6.6). Moreover, $z$ is the «-radical and $\mathbf{1}-z$ is the dual «-radical (Theorem 6.5). Thus each factor only has two RR-order: "= " and " $\leq$ ". In addition to them, type $\mathrm{I}_{\infty}$ and $\mathrm{II}_{\infty}$ factors only have one transitive HH-relation (Theorem 7.8). 
In Section 2 we provide some information about $\mathbf{H}$ - and dual $\mathbf{H}$-relations in lattices and about $\mathbf{R}$ - and dual $\mathbf{R}$-orders and their radicals. In Sections 3 and 4 we consider trails in lattices and study HH-relations and their link with trails. We prove that, if $M$ is finite then all HH-relations are generated by trails. In Section 5, for non-finite algebras, we construct $\mathbf{H H}$-relations associated with trails but not generated by trails. In Section 6 we investigate transitive HH-relations and $\mathbf{R R}$-orders and in Section 7 we describe all HH-relations in $Q$ for countably decomposable factors.

\section{Relations and radicals in complete lattices $(Q, \ll)$}

Amitsur [A1] proved that conditions $(1.1)$ and $(1.2)$ in $(Q, \ll)$ are equivalent to the conditions

$\ll$ is an H-relation if and only if $a \ll b$ implies $a \vee x \ll b \vee x$ for all $x \in Q$;

$\ll$ is a dual H-relation if and only if $a \ll b$ implies $a \wedge x \ll b \wedge x$ for all $x \in Q$.

He also introduced a procedure for construction $\mathbf{R}$-orders (resp. dual $\mathbf{R}$-orders) from $\mathbf{H}$-relations (resp. dual $\mathbf{H}$-relations). This procedure was refined in [KST1]. We sketch it below.

Consider relations $\ll^{\triangleleft}$ and $\ll^{\triangleright}$ in $Q$ defined as follows. For $a \leq b$ in $Q$, write $a \ll^{\triangleleft} b$ if there is a totally ordered set $\left(x_{\lambda}\right)_{1 \leq \lambda \leq \gamma}$ in $Q$ such that $b=x_{1}, a=x_{\gamma}, x_{\lambda+1} \ll x_{\lambda}$ for all $\lambda<\gamma$, and $x_{\beta}=\wedge_{\lambda>\beta}\left(x_{\lambda}\right)$ for all limit ordinals $\beta$.

We write $a \ll b$ if there is a totally ordered set $\left(x_{\lambda}\right)_{1 \leq \lambda \leq \gamma}$ in $Q$ such that $a=x_{1}, b=x_{\gamma}$, $x_{\lambda} \ll x_{\lambda+1}$ for all $\lambda<\gamma$, and $x_{\beta}=\vee_{\lambda<\beta}\left(x_{\lambda}\right)$ for all limit ordinals $\beta$.

Following [A1], define the lower and upper complement relations $\gtreqless$ and $\overleftarrow{\ll}$ by

$$
a \overleftarrow{\ll} b \text { if }[a, \ll] \cap[a, b]=\{a\} ; \text { and } a \gtrless b \text { if }[\ll, b] \cap[a, b]=\{b\} \text { for } a \leq b,
$$

where $[a, b]=\{z \in Q: a \leq z \leq b\}$. They are naturally linked with the relations $\ll^{\triangleleft}, \ll^{\triangleright}([\mathrm{KST} 1])$ :

$$
\overleftarrow{(\overleftrightarrow{\ll})}=\ll^{\triangleleft} \text { and } \overline{(\overleftarrow{\ll})}=\ll^{\triangleright}
$$

inf Theorem 2.1 ([KST1]) (i) Let $\ll$ be an $\mathbf{H}$-relation on $Q$. Then $\ll^{\triangleright}$ is an $\mathbf{R}$-order and $\overleftarrow{\ll}=\overleftarrow{\ll^{\triangleright}}$ is a dual $\mathbf{R}$-order. Moreover, $\ll$ is an $\mathbf{R}$-order if and only if $\ll=\ll^{\triangleright}$.

(ii) Let $\ll$ be a dual $\mathbf{H}$-relation on $Q$. Then $\ll^{\triangleleft}$ is a dual $\mathbf{R}$-order and $\vec{\ll}=\vec{\ll}$ is an $\mathbf{R}$-order. Moreover, $\ll$ is a dual $\mathbf{R}$-order if and only if $\ll=\ll^{\triangleleft}$.

An element $\mathfrak{r} \in Q$ is a $\ll$-radical and an element $\rho \in Q$ is a dual $\ll$-radical if

$$
\mathbf{0} \ll \mathfrak{r} \overleftarrow{\ll} \mathbf{1} \text { and } \mathbf{0} \ll \rho \ll \mathbf{1}
$$

The set of radicals may be empty or may have many elements. If $\ll$ is an $\mathbf{R}$-order then

$$
\mathfrak{r}=\vee[\mathbf{0}, \ll] \text { is a unique } \ll \text {-radical in } Q \text { and }[\ll, \mathfrak{r}]=[\mathbf{0}, \mathfrak{r}] \text {. }
$$

If $\ll$ is a dual $\mathbf{R}$-order then

$$
\rho=\wedge[\ll, \mathbf{1}] \text { is a unique dual } \ll \text {-radical in } Q \text { and }[\rho, \ll]=[\rho, \mathbf{1}] \text {. }
$$




\section{Cones in the lattices of all projections of $\mathrm{W}^{*}$-algebras}

Let $M$ be a $\mathrm{W}^{*}$-algebra. The set $Q=P(M)$ of all projections in $M$ with the order $\leq-$ the inclusion of the ranges of projections, is a complete lattice (Proposition 1.10.2 [Sa]).

Recall that $p, q \in Q$ are equivalent $(p \sim q)$ if there is a partial isometry $v \in M$ such that $v v^{*}=p$ and $v^{*} v=q$, so that $p v=v=v q$. We write $p^{\perp}$ for $\mathbf{1}-p$ and $p \precsim q$ if there is $r \leq q$ such that $p \sim r$. If $p \sim q$ and

$$
r \leq q \text { then } s:=v r v^{*} \in Q \text { satisfies } s \sim r \text { and } s \leq p .
$$

Note (see p. $79[\mathrm{Sa}]$ ) that if $p_{i} \sim q_{i}$ for $i=1,2, p_{1} \perp p_{2}, q_{1} \perp q_{2}$ then

$$
p_{1} \oplus p_{2} \sim q_{1} \oplus q_{2} \text {. }
$$

Recall that the central carrier $c(p)$ of $p$ is the smallest central projection majorizing $p \in Q$. Then

$$
p \sim q \text { implies } c(p)=c(q) .
$$

For $p \leq q$, let $[p, q]=\{r \in Q: p \leq r \leq q\}$. Let $M^{\prime}$ be the commutant of $M$ and $\mathfrak{Z}=M \cap M^{\prime}$ be its centre. Recall (see (1.4)) that $K \subseteq Q$ is a trail if $q \precsim p \in K$ implies $q \in K$. It is easy to see that

$$
Q z=[\mathbf{0}, z] \text { is a trail for each central projection } z \in Q \cap \mathfrak{Z} \text {. }
$$

For $p \in Q$, we call the set $\omega=\{q \in Q: q \sim p\}$ an orbit and write $\omega \precsim \omega^{\prime}$, if $p \precsim p^{\prime}$ for some $p \in \omega$ and $p^{\prime} \in \omega^{\prime}$. Orbits either coincide or do not intersect. The relation $\precsim$ does not depend on the choice of the projections in the orbits. It is reflexive, transitive and anti-symmetric. If $\omega \sim \omega^{\prime}$ then $\omega=\omega^{\prime}$. Denote by $\Omega$ the set of all orbits in $Q$. With each orbit $\omega \in \Omega$, associate the trail

$$
K_{\omega}=\cup\left\{\omega^{\prime}: \omega^{\prime} \precsim \omega\right\} .
$$

Denote by $\mathcal{K}_{M}$ the set of all trails in $Q$. Each $K \in \mathcal{K}_{M}$ has form

$$
K=K_{\Phi}=\cup_{\omega \in \Phi} K_{\omega} \text {, where } \Phi \text { is a subset of } \Omega .
$$

L6.2 Proposition 3.1 For a trail $K$ in $Q$, let $z_{K}=\vee K$ be its unique least upper bound. Set

$$
K^{\perp}=\{p \in Q:[\mathbf{0}, p] \cap K=\{\mathbf{0}\}\} .
$$

(i) $z_{K} \in \mathfrak{Z}$ and $K \subseteq\left[\mathbf{0}, z_{K}\right]$. For each $\mathbf{0} \neq p \leq z_{K}$, there is $r \in K$ such that $\mathbf{0} \neq r \leq p$.

(ii) $K^{\perp}=\left[\mathbf{0}, z_{K}^{\perp}\right]$ for $z_{K}^{\perp}=\mathbf{1}-z_{K}$.

Proof. (i) Clearly, $K \subseteq\left[\mathbf{0}, z_{K}\right]$. Let $q \in K$. For any unitary $v \in M$, we have $q \sim v^{*} q v$, since $(q v)(q v)^{*}=q$ and $(q v)^{*}(q v)=v^{*} q v$. Thus $v^{*} q v \in K$. Hence, as $z_{K}$ is an upper bound of $K, v^{*} z_{K} v$ is also an upper bound of $K$. As $z_{K}$ is the least upper bound of $K, z_{K} \leq v^{*} z_{K} v$. Then $v z_{K} v^{*} \leq v\left(v^{*} z_{K} v\right) v^{*}=z_{K}$. Thus $z_{K}=v^{*} z_{K} v$ for all unitary $v \in M$. So $z_{K} \in M^{\prime}$. Hence $z_{K} \in \mathfrak{Z}$.

Let $\mathbf{0} \neq p \leq z_{K}$. As $K \subseteq\left[\mathbf{0}, z_{K}\right]$, replace $M$ by the $\mathrm{W}^{*}$-algebra $M z_{K}=\left[\mathbf{0}, z_{K}\right]$. Thus we can assume that $\vee K=\mathbf{1}$. Let $q \in K$. By Comparability theorem [Sa, 2.1.3],

$$
q z \succsim p z \text { and } q z^{\perp} \precsim p z^{\perp} \text { for some } z \in Q \cap \mathfrak{Z} \text {. }
$$

As $q z \leq q \in K$, we have from (1.4) that $q z \in K$. As $q z \succsim p z$, it follows from (1.4) that $p z \in K$. 
If $p z \neq \mathbf{0}$ for some $q \in K$, set $r=p z$. Then $r \in K$ and $\mathbf{0} \neq r \leq p$.

Let now $p z=\mathbf{0}$ for all $q \in K$. By (3.8), $q z^{\perp} \precsim p$. If $q z^{\perp} \neq \mathbf{0}$ for some $q \in K$, there is $r \leq p$ such that $r \sim q z^{\perp} \in K$. Thus $r \in K$ and $\mathbf{0} \neq r \leq p$.

Suppose now that, for all $q \in K, p z=\mathbf{0}$ and $q z^{\perp}=\mathbf{0}$. Then $q=q z$, so that $q p=q z p=\mathbf{0}$ for all $q \in K$. Hence $q p^{\perp}=q$. Thus $q \leq p^{\perp}$, so that $p^{\perp}$ is an upper bound of $K$. As $\vee K=\mathbf{1}$ is the least upper bound of $K$, we have $p=\mathbf{0}$, a contradiction. Hence the last case impossible and there is $r \in K$ such that $\mathbf{0} \neq r \leq p$.

(ii) As $K \subseteq\left[\mathbf{0}, z_{K}\right]$, we have $\left[\mathbf{0}, z_{K}^{\perp}\right] \subseteq K^{\perp}$. Let $p \in K^{\perp}$. Suppose that $p z_{K} \neq \mathbf{0}$. Then $p z_{K} \leq z_{K}$. By (i), there is $r \in K$ such that $\mathbf{0} \neq r \leq p z_{K}$. Hence $r \leq p$, so that $r \in[\mathbf{0}, p] \cap K$, a contradiction. Thus $p z_{K}=\mathbf{0}$, so that $p \in\left[\mathbf{0}, z_{K}^{\perp}\right]$. Hence $K^{\perp} \subseteq\left[\mathbf{0}, z_{K}^{\perp}\right]$. Thus $K^{\perp}=\left[\mathbf{0}, z_{K}^{\perp}\right]$.

C3.3 Corollary 3.2 Let $K$ be a trail and $z_{K}=\vee K$. Then $K^{\perp \perp}=\left[\mathbf{0}, z_{K}\right]$ and $K^{\perp \perp \perp}=K^{\perp}=\left[\mathbf{0}, z_{K}^{\perp}\right]$.

For a trail $K$, set $K^{c}=\{p \in K: c(p) \in K\}$. A trail $K$ is complete if $K^{c}=K$.

C6.3 Lemma 3.3 Let $K$ be a trail and $z \in Q \cap \mathfrak{Z}$. Then $K z=\{p z: p \in K\}$ is a subtrail of $K$ and $K^{c}=\cup_{z \in K \cap \mathbf{3}}[\mathbf{0}, z]$. The set $K^{c}$ is the largest complete subtrail of $K$.

Proof. Let $p \in K$. As $p z \leq p$ and $K$ is a trail, $[\mathbf{0}, p z] \subseteq K$. Hence $[\mathbf{0}, p z] \subseteq K z \subseteq K$. If $q \sim p z$ then $q \in K$. By (3.3), $q \leq c(q)=c(p z) \leq z$. So $q=q z \in K z$. Thus $K z$ is a subtrail of $K$.

By (3.4), $[\mathbf{0}, z]$ is a subtrail of $K$ for each $z \in K \cap \mathfrak{Z}$. Hence $K^{c}=\cup_{z \in K \cap \mathfrak{z}}[\mathbf{0}, z]$ is a subtrail of $K$. If $p \in K^{c}$ then $p \in[\mathbf{0}, z]$ for some $z \in K \cap \mathfrak{Z}$, so that $c(p) \leq z$. Thus $c(p) \in K^{c}$. So $K^{c}$ is complete. Let $p \in K$ and $c(p) \in K$. Then $p \leq c(p) \in K \cap \mathfrak{Z}$, so that $p \in \cup_{z \in K \cap \mathfrak{z}}[\mathbf{0}, z]$. Thus $K^{c}$ is the largest complete subtrail of $K$.

By Proposition 3.1, each trail $K$ in $Q=P(M)$ gives the following decomposition of $M$.

C3.4 Corollary 3.4 Let $K \in \mathcal{K}_{M}$, let $z_{K}=\vee K$ and $z_{K^{c}}=\vee K^{c}$. Then $z_{K}, z_{K^{c}} \in Q \cap \mathfrak{Z}$. Each $\mathbf{0} \neq p \leq z_{K}$ contains $\mathbf{0} \neq r \in K, P\left(M z_{K}\right)=\left[\mathbf{0}, z_{K}\right]$ and $P\left(M z_{K}^{\perp}\right)=K^{\perp}=\left[\mathbf{0}, z_{K}^{\perp}\right]$, and

$$
M=M z_{K^{c}} \oplus M\left(z_{K} \ominus z_{K^{c}}\right) \oplus M z_{K}^{\perp} .
$$

Recall that $q \in Q$ is infinite if $p \sim q$ for some $p<q$; otherwise, $q$ is finite. It is purely infinite if it does not majorize finite projections. If $q$ is finite and $p \precsim q$ then $p$ is finite. So the set $K_{\mathrm{f}}$ of all finite projections in $Q$ is a trail. Similarly, the set $K_{\mathrm{p}}$ of all pure infinite projections in $Q$ is a trail.

By (3.7), $K_{\mathrm{f}} \subseteq K_{\mathrm{p}}^{\perp}$ and $K_{\mathrm{p}}=K_{\mathrm{f}}^{\perp}$. Thus $K_{\mathrm{p}}$ and $K_{\mathrm{f}}^{\perp \perp}$ are complete by Lemma 3.3, while $K_{\mathrm{f}}$ is not, generally, complete. Set $z_{\mathrm{f}}=\vee K_{\mathrm{f}}, z_{\mathrm{f}}^{c}=\vee K_{\mathrm{f}}^{c}, z_{\mathrm{p}}=\vee K_{\mathrm{p}}$. By Proposition 3.1, $z_{\mathrm{p}}=z_{\mathrm{f}}^{\perp}$. It is easy to see that $z_{\mathrm{f}}^{c}$ is the unique maximal finite central projection and the central projection $z_{\mathrm{sf}}=z_{\mathrm{f}}-z_{\mathrm{f}}^{c}$ is semi-finite. As $\mathbf{1}=z_{\mathrm{f}}^{c}+z_{\mathrm{sf}}+z_{\mathrm{p}}$, (3.9) gives the standard decomposition $M=M z_{\mathrm{f}}^{c} \oplus M z_{\mathrm{sf}} \oplus M z_{\mathrm{p}}$ in the sum of a finite, a properly infinite and semi-finite and a purely infinite $\mathrm{W}^{*}$-algebras.

By Proposition 2.2.8 [Sa], the set $A$ of all abelian projections in $Q$ is a trail. The subtrail $A^{c}$ consists of all central abelian projections and $z_{A^{c}}$ is the maximal central abelian projection. By (3.9), $M=M z_{A^{c}} \oplus M\left(z_{A} \ominus z_{A^{c}}\right) \oplus M z_{A}^{\perp}$, where $M z_{A^{c}}$ is the maximal commutative ideal of $M, M\left(z_{A} \ominus z_{A^{c}}\right)$ has no central abelian projections but each projection in it contains an abelian projection and $M z_{A}^{\perp}$ has no abelian projections.

Let $K$ be the trail of all minimal projections in $Q$. It is easy to see that $z_{K}=\oplus_{p \in K} c(p)$, $K^{c}=K \cap \mathfrak{Z}$ and all $p \in K^{c}$ are mutually orthogonal. Hence $z_{K^{c}}=\oplus_{p \in K \cap \mathfrak{Z}} p$. By $(3.9), M=$ $M z_{K^{c}} \oplus M\left(z_{K} \ominus z_{K^{c}}\right) \oplus M z_{K}^{\perp}$, where $P\left(M z_{K^{c}}\right)$ consists of all central minimal projections, $M\left(z_{K} \ominus z_{K^{c}}\right)$ has no central minimal projections but each projection in it majorizes a minimal projection and $M z_{K}^{\perp}$ has no minimal projections. 


\section{$4 \quad$ HH-relation in $Q=P(M)$ generated by trails}

Let $S$ be a subset in $Q$ and $\mathbf{0} \in S$. Define the reflexive relation $\ll_{S}$ on $Q$ by the condition

$$
p \ll_{S} q \text { if } p \leq q \text { and } q-p \in S .
$$

Conversely, for a reflexive relation $\ll$ in $Q$, consider the sets

$S^{\ll}=\{s \in Q: s=q-p$ for some $p \ll q\}$ and $K^{\ll}=\left\{r \in Q: r \sim s\right.$ for some $\left.s \in S^{\ll}\right\}$.

Note that (see Proposition 2.5.3 and p. $111[\mathrm{KR}]$ vol. 1)

$$
\begin{aligned}
p \wedge e & =p e \text { if } p, e \in Q \text { commute, } \\
(p \vee q)^{\perp} & =p^{\perp} \wedge q^{\perp} \text { and }(p \wedge q)^{\perp}=p^{\perp} \vee q^{\perp} \text { for } p, q \in Q .
\end{aligned}
$$

Let $p, q, r, e \in Q$. We will later use the following formulas

$$
\begin{aligned}
p e \wedge q e & =(p \wedge q) e=e(p \wedge q), \text { if } e \in Q \text { commutes with } p \text { and } q, \\
(p \oplus s) \vee(q \oplus r) & =(p \vee q) \oplus(s \vee r) \text { if } p \perp r, p \perp s, q \perp r, q \perp s ; \\
(p \oplus s) \wedge(q \oplus r) & =(p \wedge q) \oplus(s \wedge r) \text { if } p \perp r, p \perp s, q \perp r, q \perp s .
\end{aligned}
$$

Indeed, $p e \wedge q e \stackrel{(4.3)}{=}(p \wedge e) \wedge(q \wedge e)=(p \wedge q) \wedge e=(p \wedge q) e=e(p \wedge q)$. So (4.5) holds.

To prove (4.6), set $a=(p \oplus s) \vee(q \oplus r)$ and $b=(p \vee q) \oplus(s \vee r)$. As $a \geq p \vee q$ and $a \geq s \vee r$, we have $a \geq b$. On the other hand, $p \oplus s \leq b$ and $q \oplus r \leq b$, so that $a \leq b$. Thus $a=b$.

To prove (4.7), note that $p \vee q \leq s^{\perp}$, as $p \leq s^{\perp}$ and $q \leq s^{\perp}$. So $s(p \vee q)=\mathbf{0}$ and $p \vee q$ commutes with $p \oplus s$. Similarly, $r(p \vee q)=\mathbf{0}$ and $p \vee q$ commutes with $q \oplus r$. Set $a=(p \oplus s) \wedge(q \oplus r)$. Then

$$
a(p \vee q) \stackrel{(4.5)}{=}[(p \oplus s)(p \vee q)] \wedge[(q \oplus r)(p \vee q)]=p \wedge q \text { and, similarly, } a(s \vee r)=s \wedge r .
$$

Set $t=(p \vee q) \oplus(s \vee r)$. Then $p \oplus s \leq t$ and $q \oplus r \leq t$. Hence $a \leq t$. Thus (4.7) holds, as

$$
a=a t=a((p \vee q) \oplus(s \vee r))=a(p \vee q) \oplus a(s \vee r) \stackrel{(4.8)}{=}(p \wedge q) \oplus(s \wedge r) .
$$

P6.1 Theorem 4.1 (i) Let $K$ be a trail in $Q$ and $z_{K}=\vee K$. Then $\ll_{K}$ is an $\mathbf{H H}$-relation, the relations $\ll_{K^{\perp}}$ and $\ll_{K}^{\triangleright}=\ll_{K}^{\triangleleft}=\ll_{\left[\mathbf{0}, z_{K}\right]}$ are $\mathbf{R R}$-orders, and

$$
\ll_{K^{\perp}}=\overleftarrow{\ll_{K}}=\overleftrightarrow{\ll_{K}^{\triangleright}} \text { and } \ll_{K^{\perp}}=\vec{\ll}_{K}=\overrightarrow{\ll_{K}^{\triangleleft}}
$$

(ii) If $\ll$ is an $\mathbf{H}$-relation then $S^{\ll}=\{s \in Q: \mathbf{1}-s \ll \mathbf{1}\}$ and $K^{\ll}$ is a trail.

(iii) If $\ll$ is a dual $\mathbf{H}$-relation then $S^{\ll}=\{s \in Q$ : $\mathbf{0} \ll s\}=[\mathbf{0}, \ll]$ and $K^{\ll}$ is a trail.

Proof. (i) Let $p \ll_{K} q$. Consider the Kaplansky Formula ([Sa])

$$
p-(p \wedge q) \sim(p \vee q)-q \text { for } p, q \in Q .
$$

Let $p \leq r \in Q$. As $p \leq q$, we have $p \leq q \wedge r$. By (4.10), $(q \vee r)-r \sim q-(q \wedge r) \leq q-p \in K$. Thus $(q \vee r)-r \in K$ by (1.4). Hence $r \ll_{K} q \vee r$. By (1.1), $\ll_{K}$ is an H-relation. 
Let now $r \leq q$. Then $r \vee p \leq q$. By (4.10), $r-(r \wedge p) \sim(r \vee p)-p \leq q-p \in K$. So $r-(r \wedge p) \in K$ by (1.4). Thus $r \wedge p \ll_{K} r$. By (1.2), $\ll_{K}$ is a dual H-relation. So $\ll_{K}$ is an HH-relation.

Let us show that $\ll_{K^{\perp}}=\overleftarrow{\ll_{K}}$. Let $p \overleftarrow{\ll}_{K} q$ for $p, q \in Q$. By $(2.3), p \leq q$ and $p$ is not $\ll_{K}$ related to any $x \in(p, q]$, i.e., all $x-p \notin K$. Hence, by (3.7), $q-p \in K^{\perp}$, so that $p \ll_{K^{\perp}} q$.

Conversely, if $p \ll_{K^{\perp}} q$ then $q-p \in K^{\perp}$, i.e., $z \notin K$ for each $z \leq q-p$. Hence $x-p \notin K$ for each $x \in(p, q]$, so that $p$ is not $\ll_{K}$ related to $x$. Thus, by $(2.3), p \overleftarrow{\ll_{K}} q$. Hence $\ll_{K^{\perp}}=\overleftarrow{\ll_{K}}$.

Similarly, $\ll_{K^{\perp}}=\vec{\ll}_{K}$. As $\ll_{K}$ is an HH-relation, the rest of (4.9) follows from Theorem 2.1.

As $\ll_{K}$ is an $\mathbf{H H}$-relation, it follows from Theorem 2.1 that $\ll_{K}^{\triangleright}$ is an $\mathbf{R}$-order and $\ll_{K}^{\triangleleft}$ is an

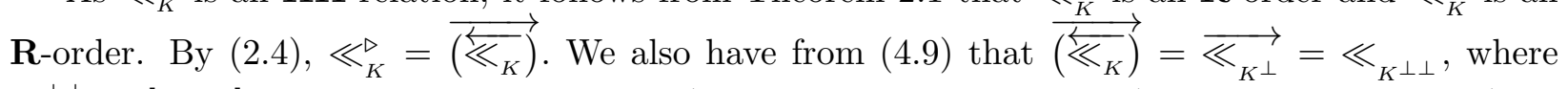
$K^{\perp \perp}=\left[\mathbf{0}, z_{K}\right]$ by Corollary 3.2. Hence $\ll_{K}^{\triangleright}=\ll_{\left[\mathbf{0}, z_{K}\right]}$. Similarly, $\ll_{K}^{\triangleleft}=\ll_{\left[\mathbf{0}, z_{K}\right]}$. Thus $\ll_{K}^{\triangleright}=$ $\ll_{K}^{\triangleleft}=\ll_{\left[0, z_{K}\right]}$ are RR-orders.

(ii) If $\ll$ is an $\mathbf{H}$-relation and $s \in S^{\ll}$, then $s=q-p$ for some $p \ll q$ in $Q$. As $p \perp q^{\perp}$,

$$
\mathbf{1}-s=p \oplus(\mathbf{1}-q) \stackrel{(4.6)}{=} p \vee q^{\perp} \stackrel{(2.1)}{\ll} q \vee q^{\perp}=\mathbf{1} .
$$

Conversely, if $\mathbf{1}-s \ll \mathbf{1}$, then $s=\mathbf{1}-(\mathbf{1}-s) \in S^{\ll}$. So $S^{\ll}=\{s \in Q: \mathbf{1}-s \ll \mathbf{1}\}$.

Let $p \leq s \in S^{\ll}$. Then $\mathbf{1}-s \ll \mathbf{1}$. As $\ll$ is an $\mathbf{H}$-relation, we get that $p \in S^{\ll}$, since $\mathbf{1}-p=$ $\begin{aligned}(\mathbf{1}-s) \oplus(s \ominus p)=(\mathbf{1}-s) \vee(s \ominus p) & \stackrel{(2.1)}{\ll} \mathbf{1} \vee(s \ominus p)=\mathbf{1} \text {. So } \\ s & \in S^{\ll} \text { implies }[\mathbf{0}, s] \in S^{\ll} .\end{aligned}$

Let $p \precsim q \in K^{\ll}$. Then $p \sim r \leq q$. By (4.2), $q \sim s$ for some $s \in S^{\ll}$. By (3.1), $r \sim a$ for some $a \leq s$. So $p \sim a$. By (4.11), $a \in S^{\ll}$. So $p \in K^{\ll}$. Thus $K^{\ll}$ is a trail.

The proof of (iii) is similar.

The next result shows the duality between $\mathbf{H H}$-relations and trails.

L6.1 Lemma 4.2 Let $K$ be a trail. Set $\ll=\ll_{K}$. Then $S^{\ll}=K^{\ll}=K$.

Proof. Let $r \in K$. For some $p \perp r$, set $q=p \oplus r$. By (4.1), $p \ll_{K} q$. Hence $r \in S^{\ll}$ by (4.2). So $K \subseteq S^{\ll}$. Conversely, let $r \in S^{\ll}$. By $(4.2), r=q-p$ for some $p \leq q$ such that $p \ll_{K} q$. So, by (4.1), $r \in K$. Thus $S^{\ll} \subseteq K$, so that $S^{\ll}=K$ is a trail. Hence $S^{\ll}=K^{\ll}=K$.

The duality between relations $\ll$ and the sets $K^{\ll}$ exists even if $\ll$ are neither $\mathbf{H}$-, nor dual H-relations and $K^{\ll}$ are not trails. For example, let $M$ be a finite $\mathrm{W}^{*}$-algebra. The relation $\ll$ in $Q$ : all $p \ll p$ and $\mathbf{0} \ll \mathbf{1}$, is neither an $\mathbf{H}$-, nor a dual $\mathbf{H}$-relation; and $S^{\ll}=K^{\ll}=\{\mathbf{0}, \mathbf{1}\}$ is not a trail. However, $\ll=\ll_{K} \ll$.

Our aim is to find conditions on an $\mathbf{H H}$-relation $\ll$ to be generated by a trail $K$ : $\ll \ll_{K}$.

L3.2 Lemma 4.3 Let $\ll$ be an $\mathbf{H H}$-relation. Then

(i) $q-p \in S^{\ll}$ for $p \leq q$, if and only if $p \ll q$;

(ii) $\ll=\ll_{S} \ll$;

(iii) $\ll=\ll_{K}$ if and only if $S^{\ll}=K^{\ll}$. 
Proof. (i) If $p \ll q$ then $q-p \in S^{\ll}$ by (4.2). Conversely, let $q-p \in S^{\ll}$. As $\mathbf{0} \ll q-p$ by Theorem 4.1(iii), we have from (1.1) and (4.6) that $p \ll(q-p) \vee p=(q-p) \oplus p=q$.

(ii) Let $p \ll q$. Then $q-p \in S^{\ll}$ by (4.2). Hence $p \ll_{S} \ll q$ by (4.1). Conversely, if $p \ll_{S} \ll q$ then $q-p \in S^{\ll}$ by (4.2). By (i), this implies $p \ll q$. Thus $\ll=\ll_{S} \ll$.

(iii) If $\ll=\ll_{K \ll}, K^{\ll}$ is a trail by Theorem 4.1(ii). By Lemma $4.2, S^{\ll}=K^{\ll}$.

Conversely, if $S^{\ll}=K^{\ll}$ then $\ll=\ll_{S} \ll \ll_{K}$ by (ii).

P6.5 Lemma 4.4 Let $\ll$ be an $\mathbf{H H}$-relation and $p, q \in Q$. Set $l=p \vee q$.

(i) If there exists $r \in Q$ such that $q \leq p \vee r$ and $q \wedge r=\mathbf{0}$, then $\mathbf{0} \ll p$ implies $\mathbf{0} \ll q$.

(ii) Let $p \perp q$ and $p \sim q$. There is $t \leq l$ such that $t \wedge p=t \wedge q=\mathbf{0}, t \sim p$ and $t \vee p=l$.

Proof. (i) By (1.1), $\mathbf{0} \ll p$ implies $r \ll p \vee r$. So $\mathbf{0}=q \wedge r \ll q \wedge(p \vee r)=q$ by (1.2).

(ii) As $p \sim q$, we have $p=v v^{*}, q=v^{*} v, p v=v=v q$. Thus $l p=p, q l=q$. So, as $p \perp q$,

$$
l v=l p v=p v=v=v q=v q l=v l, v^{2}=v q p v=\mathbf{0}, v^{*} l=v^{*}=l v^{*} \text { and } v^{*} v^{*}=\mathbf{0} .
$$

Let $t=\frac{1}{2}\left(l+v+v^{*}\right)$. Then $t$ is a projection and $t \leq l$, since $t^{*}=t$,

$$
t^{2}=\frac{1}{4}\left(l+v l+v^{*} l+l v+v^{2}+v^{*} v+l v^{*}+v v^{*}+v^{*} v^{*}\right)=t \text { and } t l=l t=t .
$$

Set $s=t \wedge q$. Then $s q=s t=s l=s$ and $s v=(s q)(p v)=\mathbf{0}$, as $p \perp q$. Hence

$$
s=s t=\frac{1}{2}\left(s l+s v+s v^{*}\right)=\frac{1}{2}\left(s+s v^{*}\right), \text { so that } s=s v^{*} \text { and } s=s q=s v^{*} v=s v=\mathbf{0} .
$$

Thus $t \wedge q=\mathbf{0}$. Similarly, $t \wedge p=\mathbf{0}$. Set $r=t \vee p$. Then $r \leq l$. Let $a=l-r$. Then $a t=a p=\mathbf{0}$ and $a l=a$. So $a q=a(l-p)=a$. Thus $a \leq q$ and $a v=a q p v=\mathbf{0}$. Hence

$$
\begin{aligned}
& \mathbf{0}=a t=\frac{1}{2}\left(a l+a v+a v^{*}\right)=\frac{1}{2}\left(a+a v^{*}\right), \text { so that } \\
& \mathbf{0}=\left(a+a v^{*}\right) p v=a v^{*} p v=a v^{*} v=a q=a .
\end{aligned}
$$

Hence $l=r=t \vee p$. Therefore, as $t \wedge q=t \wedge p=\mathbf{0}$ and $l=p \oplus q$, we have from (4.10) that $t=t-t \wedge p \sim(t \vee p)-p=l-p=q \sim p$ which completes the proof.

We will now consider cases when $\mathbf{0} \ll p$ and $p \sim q$ imply $\mathbf{0} \ll q$.

P4.2 Proposition 4.5 Let $\ll$ be an $\mathbf{H H}$-relation in $Q$. If $p \perp q$ and $p \sim q$ then $\mathbf{0} \ll p$ implies $\mathbf{0} \ll q$.

Proof. As $\mathbf{0} \ll p$ and $\ll$ is a $\mathbf{H}$-relation, $q \ll p \vee q=l$ by (1.1). Let $t \in Q$ be as in Lemma 4.4. Then, as $\ll$ is a dual H-relation, $\mathbf{0}=q \wedge t \ll l \wedge t=t$ by (2.2). Hence $p=\mathbf{0} \vee p \ll t \vee p=l$ by (1.1). So $\mathbf{0}=p \wedge q \ll l \wedge q=q$ by (2.2).

For $p, q \in Q$, set $p_{0}=p \ominus(p \wedge q) \ominus\left(p \wedge q^{\perp}\right)$ and $q_{0}=q \ominus(p \wedge q) \ominus\left(p^{\perp} \wedge q\right)$. Let $b=p_{0} \vee q_{0}$ and $M_{0}$ be the $\mathrm{W}^{*}$-algebra generated by $p_{0}, q_{0}$. Then ([T], p. 306)

$$
\begin{aligned}
p & =p_{0} \oplus(p \wedge q) \oplus\left(p \wedge q^{\perp}\right) \text { and } q=q_{0} \oplus(p \wedge q) \oplus\left(p^{\perp} \wedge q\right), \\
\mathbf{1} & =b \oplus(p \wedge q) \oplus\left(p \wedge q^{\perp}\right) \oplus\left(p^{\perp} \wedge q\right) \oplus\left(p^{\perp} \wedge q^{\perp}\right), \\
p_{0} \wedge q_{0} & =p_{0} \wedge\left(b \ominus q_{0}\right)=\left(b \ominus p_{0}\right) \wedge q_{0}=\mathbf{0} \text { and } p_{0} \sim q_{0} \sim b \ominus p_{0} \sim b \ominus q_{0} \text { in } M_{0} .
\end{aligned}
$$


P3.3 Proposition 4.6 Let $\ll$ be an $\mathbf{H H}$-relation and $p$ be finite. If $\mathbf{0} \ll p$ and $p \sim q$ then $\mathbf{0} \ll q$.

Proof. If $p^{\perp} \wedge q=\mathbf{0}$ then, setting $r=p^{\perp}$ in Lemma 4.4(i), we have $\mathbf{0} \ll q$.

Assume now that $p^{\perp} \wedge q \neq \mathbf{0}$. Consider the representation of $p$ and $q$ given in (4.12). Set

$$
p_{1}=p_{0} \oplus(p \wedge q) \text { and } q_{1}=q_{0} \oplus(p \wedge q) .
$$

As $p_{0} \sim q_{0}$ in $M\left(p_{0}, q_{0}\right)$, we have $p_{1} \sim q_{1}$. As $p$ is finite, $q$ is finite. Thus $p \vee q$ is finite by Theorem V.1.37 [T]. Hence the $\mathrm{W}^{*}$-algebra $M_{1}=(p \vee q) M(p \vee q)$ is finite and $p \sim q$ and $p_{1} \sim q_{1}$ in $M_{1}$.

Set $p_{2}=p-p_{1}, q_{2}=q \ominus q_{1}$. Then $p_{2}=p \wedge q^{\perp}, q_{2}=p^{\perp} \wedge q \neq \mathbf{0}$ and it follows from Proposition 2.4.2 [Sa] that they are equivalent: $p_{2}=v v^{*}$ and $q_{2}=v^{*} v$ for a partial isometry $v$. They are also orthogonal. Set $l=p_{2} \oplus q_{2}$ and $t=\frac{1}{2}\left(l+v+v^{*}\right)$. Replacing $p$ by $p_{2}$ and $q$ by $q_{2}$ in Lemma 4.4(ii), we obtain from its proof that

$$
t \text { is a projection, } t \leq l, q_{2} \wedge t=\mathbf{0} \text { and } l=p_{2} \vee t .
$$

We have $p=p_{0} \oplus(p \wedge q) \oplus p_{2}$ and $q=q_{0} \oplus(p \wedge q) \oplus q_{2}$. Set $s=b \ominus p_{0}$. By (4.12), $b=p_{0} \vee q_{0}$ is orthogonal to $l=p_{2} \oplus q_{2}$, so that $s \perp l$. Moreover, $s \perp\left((p \wedge q) \oplus p_{2}\right), t \perp p_{0}$ and $t \perp(p \wedge q)$. Hence, by (4.12) and (4.6),

$$
\begin{aligned}
p \vee(s \oplus t) & =\left(p_{0} \oplus(p \wedge q) \oplus p_{2}\right) \vee(s \oplus t)=\left(p_{0} \vee s\right) \oplus\left(\left((p \wedge q) \oplus p_{2}\right) \vee t\right) \\
& =\left(p_{0} \vee s\right) \oplus(p \wedge q) \oplus\left(p_{2} \vee t\right) \stackrel{(4.13)}{=}\left(p_{0} \vee q_{0}\right) \oplus(p \wedge q) \oplus l \geq q
\end{aligned}
$$

Similarly, by (4.12), $s \perp\left((p \wedge q) \oplus q_{2}\right), t \perp q_{0}$ and $t \perp(p \wedge q)$. Hence, by (4.7),

$$
\begin{aligned}
q \wedge(s \oplus t) & \left.=\left(q_{0} \oplus(p \wedge q) \oplus q_{2}\right) \wedge(s \oplus t)=\left(q_{0} \wedge s\right) \oplus\left((p \wedge q) \oplus q_{2}\right) \wedge t\right) \\
& =\left(q_{0} \wedge s\right) \oplus\left(q_{2} \wedge t\right) \stackrel{(4.13)}{=} q_{0} \wedge s \leq q_{0} \wedge\left(b \ominus p_{0}\right) \stackrel{(4.12)}{=} \mathbf{0} .
\end{aligned}
$$

Setting $r=s \oplus t$ in Lemma 4.4(i), we get $\mathbf{0} \ll q$.

Denote by $F$ the trail of all finite projections in $Q=P(M)$ and by $\mathbf{H H}_{\text {rel }}$ the set of all $\mathbf{H H}$ relations. It follows from Theorem 4.1 that

$$
\theta: \ll \rightarrow K^{\ll} \text { maps } \mathbf{H H}_{\text {rel }} \text { to } \mathcal{K}_{M} \text { and } \phi: K \rightarrow \ll_{K} \text { maps } \mathcal{K}_{M} \text { to } \mathbf{H H}_{\text {rel }} \text {. }
$$

C3.0 Corollary 4.7 (i) $S^{\ll} \cap F=K^{\ll} \cap F$ is a trail for each $\mathbf{H H}$-relation $\ll$ in $Q$.

(ii) $\phi$ is an injective map from $\mathcal{K}_{M}$ to $\mathbf{H H}_{\mathrm{rel}}$ and $\theta \circ \phi=\mathbf{1}_{\mathcal{K}_{M}}$.

(iii) If $M$ is a finite $W^{*}$-algebra then $S^{\ll}=K^{\ll}$ and $\ll=\ll_{K} \ll$ for each $\mathbf{H H}$-relation $\ll$.

Proof. (i) If $p \precsim q \in S^{\ll} \cap F$ then $p \sim r \leq q$ for some $r \in Q$. By Theorem 4.1(ii), $r \in S^{\ll}$. As $q$ is finite, $r$ is finite. As $p \sim r, p$ is finite and $p \in S^{\ll}$ by Proposition 4.6. Thus $p \in S^{\ll} \cap F$, so that $S^{\ll} \cap F$ is a trail and $S^{\ll} \cap F=K^{\ll} \cap F$ by (4.2).

Part (ii) follows from Lemma 4.2. Part (iii) follows from (i) and Lemma 4.3(iii), as $F=Q$.

Corollary 4.7 yields

T3.1 Corollary 4.8 If $M$ is a finite $W^{*}$-algebra then all $\mathbf{H H}$-relations in $Q=P(M)$ are generated by trails, the map $\phi$ is an isomorphism from $\mathcal{K}_{M}$ onto $\mathbf{H H}_{\mathrm{rel}}$ and $\theta=\phi^{-1}$. 


\section{HH-relations in $P(M)$ not generated by trails}

If $M$ is not finite then, apart from $\mathbf{H H}$-relations generated by trails in $P(M)$, there are also $\mathbf{H H}$ relations associated but not generated by trails, i.e., $\phi\left(\mathcal{K}_{M}\right) \varsubsetneqq \mathbf{H H}_{\text {rel }}$. In this section we consider such relations.

C3,0 Corollary 5.1 Let $M$ be not finite and let $\ll$ be an $\mathbf{H H}$-relation in $Q$ not generated by a trail. Then $S^{\ll}$ contains infinite projections. If $p \precsim s \in S^{\ll}$ and $p$ is finite then $p \in S^{\ll}$.

Proof. If $S^{\ll}=S^{\ll} \cap F$ then $S^{\ll}$ is a trail by Corollary 4.7(i). So $S^{\ll}=K^{\ll}$ by (4.2). By Lemma $4.3, \ll=\ll_{K}$, i.e., $\ll$ is generated by a trail, a contradiction. Thus $S^{\ll}$ has infinite projections. Furthemore, if $p \precsim s \in S^{\ll}$ and $p \in F$, there is a finite $r \leq s$ such that $p \sim r$. By Theorem 4.1(ii), $r \in S^{\ll} \cap F$. As $S^{\ll} \cap F$ is a trail, $p \in S^{\ll}$.

Let $M$ be a $\mathrm{W}^{*}$-algebra of operators on a Hilbert space $H$. For $p \in Q$, let $p^{\perp}=\mathbf{1}-p$ and $H_{p}=p H$ be the range of $p$ in $H$.

P5.1 Proposition 5.2 Let $p \leq q$ in $Q$. Then, for each $r \in Q$,

$$
\begin{aligned}
& (q-p)^{\perp} \leq(q \vee r-p \vee r)^{\perp} ; \\
& (q-p)^{\perp} \precsim(q \wedge r-p \wedge r)^{\perp} .
\end{aligned}
$$

Proof. We have $H_{q}+H_{r}=H_{q} \oplus q^{\perp} H_{r}$. Hence $H_{q \vee r}=\overline{H_{q}+H_{r}}=\overline{H_{q} \oplus q^{\perp} H_{r}}=H_{q} \oplus \overline{q^{\perp} H_{r}}$. Thus $s:=(q \vee r)-q$ is the projection on $\overline{q^{\perp} H_{r}}$. Similarly, $t:=(p \vee r)-p$ is the projection on $\overline{p^{\perp} H_{r}}$. As $p \leq q$, we have $q^{\perp} \leq p^{\perp}$. Thus $\overline{q^{\perp} H_{r}} \subseteq \overline{p^{\perp} H_{r}}$. Hence $s \leq t$.

Set $\lambda=q \vee r-p \vee r$. It is a projection, since $q \vee r \geq p \vee r$. Set $a=q-p, b=t-s$. Then $\lambda=(q+s)-(p+t)=a-b$. As $\lambda^{2}=\lambda$, we have $2 b=a b+b a$. Multiplying by $a$, we get $2 a b=a b+a b a$, so that $a b=a b a$. Hence $a b=b a$. So $b=a b=b a$. Thus $b \leq a$. Therefore $\lambda \leq a$, i.e., $(q \vee r-p \vee r) \leq q-p$. So (5.1) holds.

To prove (5.2), set $s=q \wedge r$. Then (5.2) becomes

$$
(q-p)^{\perp} \precsim(s-p \wedge s)^{\perp}, \text { where } s \leq q .
$$

We have $(q-p)^{\perp}=q^{\perp} \oplus p$ and

$$
(s-p \wedge s)^{\perp}=(q \ominus(q \ominus(s-p \wedge s)))^{\perp}=q^{\perp} \oplus((q \ominus s) \oplus(p \wedge s)) .
$$

Therefore in order to prove (5.3) it suffices to show that

$$
p \precsim((q \ominus s) \oplus(p \wedge s)) .
$$

As $s \leq q$ and $p \leq q$, consider the $\mathrm{W}^{*}$-algebra $M_{q}=q M q$ with $\mathbf{1}:=\mathbf{1}_{M_{q}}=q$. Then $q \ominus s=s^{\perp}$ and to prove (5.4), we have to show that

$$
p \precsim\left(s^{\perp} \oplus(p \wedge s)\right) \text { in } M_{q} .
$$

Replacing $q$ by $s$ in (4.12), we have that there $p_{0} \leq p$ and $s_{0} \leq s$ such that

$$
\begin{aligned}
& p=p_{0} \oplus(p \wedge s) \oplus\left(p \wedge s^{\perp}\right) \text { and } s=s_{0} \oplus(p \wedge s) \oplus\left(p^{\perp} \wedge s\right), \\
& \mathbf{1}=b \oplus(p \wedge s) \oplus\left(p \wedge s^{\perp}\right) \oplus\left(p^{\perp} \wedge s\right) \oplus\left(p^{\perp} \wedge s^{\perp}\right) \text { and } p_{0} \sim b \ominus s_{0},
\end{aligned}
$$


where $b=p_{0} \vee s_{0}$. Then $s^{\perp}=\mathbf{1}-s=\left(b \ominus s_{0}\right) \oplus\left(p \wedge s^{\perp}\right) \oplus\left(p^{\perp} \wedge s^{\perp}\right)$. As $p_{0} \sim b \ominus s_{0}$,

$$
\begin{aligned}
p & =\left(p_{0} \oplus(p \wedge s) \oplus\left(p \wedge s^{\perp}\right)\right) \sim\left(\left(b \ominus s_{0}\right) \oplus(p \wedge s) \oplus\left(p \wedge s^{\perp}\right)\right) \\
& \leq\left(\left(b \ominus s_{0}\right) \oplus(p \wedge s) \oplus\left(p \wedge s^{\perp}\right) \oplus\left(p^{\perp} \wedge s^{\perp}\right)\right)=\left(s^{\perp} \oplus(p \wedge s)\right),
\end{aligned}
$$

so that (5.5) holds which completes the proof of (5.2).

For a trail $K \in \mathcal{K}_{M} \backslash Q$, define the relation $\ll_{\perp}^{K}$ in $Q$ :

$$
p \ll_{\perp}^{K} q \text { if } p \leq q \text { and }(q-p)^{\perp} \notin K .
$$

T5.2 Theorem 5.3 (i) For each trail $K \in \mathcal{K}_{M} \backslash Q, \ll_{\perp}^{K}$ is an $H H$-relation in $Q=P(M)$ and

$$
S^{\ll}{ }_{\perp}^{K}=\left\{s \in Q: \mathbf{0} \ll_{\perp}^{K} s\right\}=\left\{s \in Q: s^{\perp} \notin K\right\}=Q \backslash\left\{q^{\perp}: q \in K\right\} .
$$

(ii) If $M$ is a finite $W^{*}$-algebra then $R:=S_{\perp}^{\ll_{\perp}^{K}}$ is a trail and $\ll_{\perp}^{K}$ is generated by $R$.

(iii) If $M$ is properly infinite then $S_{\perp}^{\ll_{\perp}^{K}}$ is not a trail and $\ll_{\perp}^{K}$ is not generated by a trail.

Proof. (i) Let $p \ll_{\perp}^{K} q$, i.e., $p \leq q$ and $(q-p)^{\perp} \notin K$. For each $r \in Q, p \vee r \leq q \vee r$ and, by (5.1), $(q-p)^{\perp} \leq(q \vee r-p \vee r)^{\perp}$. If $(q \vee r-p \vee r)^{\perp} \in K$ then, as $K$ is a trail, $(q-p)^{\perp} \in K$ by (1.4), a contradiction. Hence $(q \vee r-p \vee r)^{\perp} \notin K$. So $p \vee r \ll_{\perp}^{K} q \vee r$ by (5.6).

Similarly, using (5.2), we get $p \wedge r \ll_{\perp}^{K} q \wedge r$. Thus, by (2.1) and (2.2), $\ll_{\perp}^{K}$ is an $H H$-relation. So (5.7) follows from (4.2), Theorem 4.1 and (5.6).

(ii) Let $M$ be finite and $r \in R$. Then $r^{\perp} \notin K$. If $p \leq r$ then $r^{\perp} \leq p^{\perp}$. If $p^{\perp} \in K$ then $r^{\perp} \in K$. This contradiction shows that $p^{\perp} \notin K$. Thus $p \in R$.

Let $q \sim r$. Since $M$ is finite, $q^{\perp} \sim r^{\perp}$ by Proposition V.1.38 [T]. As $K$ is a trail and $r^{\perp} \notin K$, we have $q^{\perp} \notin K$. Thus $q \in R$. So $R$ is a trail by (1.4). By Lemma 4.3(ii), $\ll_{\perp}^{K}=\ll_{R}$.

(iii) Let $M$ be properly infinite, i.e., all $z \in Q \cap \mathfrak{Z}$ are infinite. Then (see Lemma 6.3.3 [KR]) there is $e \in Q$ such that $e \sim e^{\perp} \sim \mathbf{1}$. If $e^{\perp} \in K$ then $\mathbf{1} \in K$ and $K=Q$, as $K$ is a trail, a contradiction. Hence $e^{\perp} \notin K$, so that $e \in S^{\ll_{\perp}^{K}}$. If $S^{\ll_{\perp}^{K}}$ is a trail then $\mathbf{1} \in S^{\ll{ }_{\perp}^{K}}$, so that $S^{\ll_{\perp}^{K}}=Q$ which is impossible. Thus $S^{\ll}{ }_{\perp}^{K}$ is not a trail.

Suppose that $\ll_{\perp}^{K}$ is generated by a trail $C$, i.e., $\ll_{\perp}^{K}=\ll_{C}$. Then $S^{\ll_{\perp}^{K}}=S^{\ll_{C}}=C$ is a trail by Lemma 4.2, a contradiction which completes the proof.

In the last section we show that if $M$ is a countably decomposable factor then each $\mathbf{H H}$-relation $\ll$ in $P(M)$ is either $\ll_{K}$, or $\ll_{\perp}^{K}$ for some trail $K$. If $M$ is not a factor then the structure of $\mathbf{H H}$ relations is much more complicated. Apart from $\ll_{K}$ and $\ll_{\perp}^{K}, K \in \mathcal{K}_{M}$, there are also many other "mixed type" HH-relations neither generated by trails, nor associated with them.

For example, let $z \in Q \cap \mathfrak{Z}$ and $M z$ be not a finite $\mathrm{W}^{*}$-algebra. Let $K_{1} \neq\{\mathbf{0}\}$ be a trail in $Q z$ and $K_{2}$ be a trail in $Q z^{\perp}$. Consider the following relation in $Q$ : for $p \leq q$,

$$
p \ll q \text { if either } p, q \in Q z \text { and } q-p \in K_{1} \text {, or } p, q \in Q z^{\perp} \text { and }(q-p)^{\perp} z^{\perp} \notin K_{2} \text {. }
$$

Then $\ll$ is an HH-relation and $S^{\ll}=K_{1} \cup S^{\ll_{\perp}^{K_{2}}}$, where $S^{\ll_{\perp}^{K_{2}}}=\left\{q \in Q z^{\perp}: q^{\perp} z^{\perp} \notin K_{2}\right\}$. By Theorem 5.3, $S_{\perp}^{K_{\perp}}$ is not a trail in $Q z^{\perp}$. Hence $S^{\ll}$ is not a trail and there does not exist a trail $R$ in $Q$ such that $S^{\ll}=S_{\perp}^{\ll}$. Thus $\ll$ is neither generated by a trail, nor associated with a trail. 


\section{$6 \quad$ HH-orders and RR-orders in the lattices of projections}

If an $\mathbf{H H}$-relation is transitive, we call it an $\mathbf{H H}$-orders. A subset $L \subseteq Q$ is a sublattice if

$$
p \wedge q \in L \text { and } p \vee q \in L \text { for } p, q \in L .
$$

P6.2 Theorem 6.1 (i) Let $\ll$ be an $\mathbf{H H}$-relation. The following are equivalent.

1) $\ll$ is transitive; $\quad 2) S \ll$ is a sublattice;

3) if $p, q \in S^{\ll}$ and $p \perp q$, then $p \oplus q \in S^{\ll}$.

(ii) If $\ll$ is an HH-order then $\mathbf{0} \ll p \sim q$ implies $\mathbf{0} \ll q$. Also $S^{\ll}=K^{\ll}$ and $\ll=\ll_{K \ll}$.

(iii) A relation $\ll$ is an $\mathbf{H H}-$ order if and only if $\ll=\ll_{K}$ and the trail $K$ is a lattice.

Proof. (i) 1) $\Rightarrow 2$ ). Let $\ll$ be transitive and $p, q \in S^{\ll}$. As $p \wedge q \leq p, p \wedge q \in S^{\ll}$ by (4.11). As $\mathbf{0} \ll q, p \ll p \vee q$ by (2.1). Since $\mathbf{0} \ll p$, we have $\mathbf{0} \ll p \vee q$. So $p \vee q \in S \ll$ and $S^{\ll}$ is a sublattice.

$2) \Rightarrow 3$ ) is evident. 3) $\Rightarrow 1$ ). If $p \ll q \ll r$ then $q-p, r-q \in S^{\ll}$ by $(4.2)$ and $(q-p) \perp(r-q)$. Hence $(r-q) \oplus(q-p)=r-p \in S^{\ll}$. By Lemma 4.3(i), $p \ll r$. So $\ll$ is transitive.

(ii) By (4.10), $q-(q \wedge p) \sim(q \vee p)-p$. As $q-(q \wedge p) \leq q \sim p$, it follows from (3.1) that $q-(q \wedge p) \sim r$ for some $r \leq p$. So $(q \vee p)-p \sim r$. As $\mathbf{0} \ll p$, we have $\mathbf{0}=\mathbf{0} \wedge r \ll p \wedge r=r$ by (2.2). Since $((q \vee p)-p) \perp p$, also $((q \vee p)-p) \perp r$. As $\mathbf{0} \ll r, \mathbf{0} \ll(q \vee p)-p$ by Proposition 4.5.

As $\ll$ is transitive, $S^{\ll}$ is a sublattice by (i). Hence $((q \vee p)-p) \vee p \stackrel{(4.6)}{=}((q \vee p)-p) \oplus p=q \vee p \in S^{\ll}$. Then $q \in S^{\ll}$ by (4.11), as $q \leq q \vee p$. Thus $S^{\ll}=K^{\ll}$ by (4.2). So $\ll=\ll_{K}$ by Lemma 4.3(iii).

(iii) If $\ll$ is an $\mathbf{H H}$-order, $\ll=\ll_{K}$ and $K^{\ll}=S^{\ll}$ is a trail by (ii). By (i), $K^{\ll}$ is a lattice. Conversely, if $\ll=\ll_{K}$ for a trail $K$, then $\ll$ is an HH-relation by Theorem 4.1(i) and $S^{\ll}=K$ by Lemma 4.2 . As $K$ is a lattice, $\ll$ is transitive by (i).

Let $\mathbf{H H}_{\text {ord }}$ be the set of all $\mathbf{H H}$-orders and $\mathcal{K}_{M}^{\text {lat }}$ the set of all trails in $P(M)$ which are lattices.

T5.1 Corollary 6.2 Let $M$ be a $W^{*}$-algebra. The map $\phi$ in (4.14) isomorphically maps $\mathcal{K}_{M}^{\text {lat }}$ onto $\mathbf{H H}_{\mathrm{ord}}$ and the restriction of the map $\theta$ in (4.14) to $\mathbf{H H}_{\mathrm{ord}}$ is its inverse.

Proof. For $K \in \mathcal{K}_{M}^{\text {lat }}, \phi(K)=\ll_{K}$ is an $\mathbf{H H}$-order by Theorem 6.1(iii). So, by Corollary 4.7(ii), $\phi$ injectively maps $\mathcal{K}_{M}^{\text {lat }}$ in $\mathbf{H H}_{\text {ord }}$.

Let $\ll$ be an $\mathbf{H H}$-order. By Theorem $6.1, \ll=\ll_{K}$, where $K$ is a trail and also a lattice. By Lemma $4.2, S^{\ll}=K^{\ll}=K$, so that $K^{\ll} \in \mathcal{K}_{M}^{\text {lat }}$ and $\phi\left(K^{\ll}\right)=\ll_{K}=\ll$. Hence $\left.\phi\right|_{\mathcal{K}_{M}^{\text {lat }}}$ is an isomorphism of $\mathcal{K}_{M}^{\text {lat }}$ onto $\mathbf{H H}_{\text {ord }}$. Combining this with Corollary 4.7, we conclude the proof.

Note that $\mathbf{R}$-orders or dual $\mathbf{R}$-orders are not necessarily $\mathbf{R R}$-orders as the example below shows.

E6.3 Example 6.3 Consider the following relation $\ll$ on $Q: p \ll p$ and $p \ll \mathbf{1}$ for all $p \in Q$. It is transitive and satisfies (2.1). For each $p \in Q$, the set $[p, \ll]=\{p, \mathbf{1}\}$ has the least upper bound $\mathbf{1}$. Thus $\ll$ is an R-order. However, it is not a dual $\mathbf{H}$-relation. Indeed, let $p<q<\mathbf{1}$. Then $p \ll \mathbf{1}$, but $p=p \wedge q \nless q$, so that (1.2) does not hold.

If, however, an $\mathbf{R}$-order is generated by a trail in $Q$ then it is also a dual $\mathbf{R}$-order.

C6.1 Corollary 6.4 Let $K$ be a trail. The following conditions are equivalent.

(i) $\ll_{K}$ is an $\mathbf{R}$-order in $Q ; \quad$ (ii) $\ll_{K}$ is a dual $\mathbf{R}$-order in $Q$;

(iii) $K=[\mathbf{0}, z]$ where $z=\vee K \in Q \cap \mathfrak{Z}$. 
Proof. (i) $\Rightarrow$ (iii). Let $\ll_{K}$ be an R-order. By Theorem 4.1(i), $\ll_{K^{\perp}}=\overleftarrow{\ll}_{K}=\overleftrightarrow{\ll_{K}}$. Thus

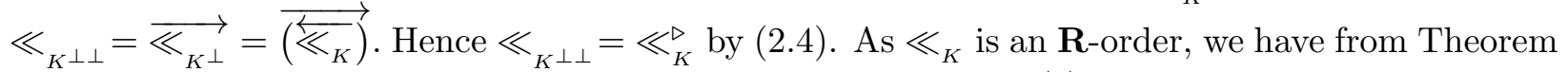
2.1(i) that $\ll_{K}=\ll_{K}^{\triangleright}$. Thus $\ll_{K \perp \perp}=\ll_{K}$. By Corollary 3.2, $K^{\perp \perp}=[\mathbf{0}, z]$. Hence $\mathbf{0} \ll_{K^{\perp \perp}} z$ by (4.1). As $\ll_{K^{\perp \perp}}=\ll_{K}$, we have $\mathbf{0} \ll_{K} z$. So $z=z-\mathbf{0} \in K$ by (4.1). As $K$ is a trail, [0, $\left.z\right] \subseteq K$. On the other hand, $K \subseteq[\mathbf{0}, z]$ by Proposition 3.1. Thus $K=[\mathbf{0}, z]$.

The proof of (ii) $\Longrightarrow$ (iii) is similar.

(iii) $\Longrightarrow$ (i), (ii). Let $K=[\mathbf{0}, z]$. By Corollary $3.2, K^{\perp \perp}=[\mathbf{0}, z]=K$. So $\ll_{K}=\ll_{K^{\perp \perp}}$ is an RR-order by Theorem 4.1(i).

T6.2 Theorem 6.5 Let $\ll$ be an $\mathbf{R R}$-order and $\mathfrak{r}=\vee[\mathbf{0}, \ll]($ see $(2.6))$ be the $\ll$-radical. Then $\mathfrak{r} \in Q \cap \mathfrak{Z}$,

$$
K^{\ll}=[\mathbf{0}, \ll]=[\mathbf{0}, \mathfrak{r}]=[\ll, \mathfrak{r}], \ll=\ll_{[\mathbf{0}, \mathfrak{r}]} \text { and } \rho=\mathbf{1}-\mathfrak{r} \text { is the dual } \ll \text {-radical. }
$$

Proof. By Theorem 6.1, $\ll=\ll_{K}$ for some trail $K$ which is a lattice. By Corollary 6.4, $K=[\mathbf{0}, z]$ where $z=\vee K \in Q \cap \mathfrak{Z}$. By Lemma $4.2, S^{\ll}=K^{\ll}=K=[\mathbf{0}, z]$. Hence we have from Theorem 4.1 that $S^{\ll}=[\mathbf{0}, \ll]=[\mathbf{0}, z]$. Thus $\mathfrak{r}=\vee[\mathbf{0}, \ll]=\vee[\mathbf{0}, z]=z$. As $[\mathbf{0}, \mathfrak{r}]=[\ll, \mathfrak{r}]$ by $(2.6)$, $K^{\ll}=[\mathbf{0}, \ll]=[\mathbf{0}, \mathfrak{r}]=[\ll, \mathfrak{r}]$ and $\ll=\ll_{[\mathbf{0}, \mathfrak{r}]}$.

As $\ll$ is a dual $\mathbf{R}$-order, $\rho=\wedge[\ll, \mathbf{1}]$ is the dual $\ll$-radical by $(2.7)$ and $\mathbf{0} \ll \rho \ll \mathbf{1}$ by $(2.5)$. As $\ll=\ll_{[\mathbf{0}, \mathfrak{r}]}$, we have $\rho \ll_{[\mathbf{0}, \mathfrak{r}]} \mathbf{1}$. So $\mathbf{1}-\rho \in[\mathbf{0}, \mathfrak{r}]$, i.e., $\mathbf{1}-\rho \leq \mathfrak{r}$. Thus $\mathbf{0} \leq \mathfrak{r}-(\mathbf{1}-\rho)=\rho-(\mathbf{1}-\mathfrak{r}) \leq \mathfrak{r}$. So $\rho-(\mathbf{1}-\mathfrak{r}) \in[\mathbf{0}, \mathfrak{r}]$. Hence $(\mathbf{1}-\mathfrak{r}) \ll_{[\mathbf{0}, \mathfrak{r}]} \rho$, so that $(\mathbf{1}-\mathfrak{r}) \ll \rho$. As $\mathbf{0} \ll \rho, \mathbf{1}-\mathfrak{r}=\rho$ by $(2.3)$.

By Corollary 6.4, the map $z \rightarrow \ll_{[\mathbf{0}, z]}$ maps the set $Q \cap \mathfrak{Z}$ into the set of all RR-orders.

C3.2 Corollary 6.6 (i) For each trail $K$, the projection $z_{K}=\vee K \in Q \cap \mathfrak{Z}$ is the $\ll_{K}^{\triangleright}$-radical and $z_{K}^{\perp}$ is the dual $\ll_{K}^{\triangleright}$-radical: $\mathbf{0} \ll_{K}^{\triangleright} z_{K} \overleftarrow{\ll_{K}^{\triangleright}} \mathbf{1}$ and $\mathbf{0} \vec{\ll}_{K}^{\gtrless_{K}} z_{K^{\perp}} \ll_{K}^{\triangleright} \mathbf{1}$.

(ii) The map $z \rightarrow \ll_{[\mathbf{0}, z]}$ is a one-to-one map from $Q \cap \mathfrak{Z}$ onto the set of all $\mathbf{R R}$-orders in $Q$.

Proof. (i) By Theorem 4.1, $\ll_{K}^{\triangleright}=\ll_{\left[\mathbf{0}, z_{K}\right]}$ is an RR-order. Hence, by Theorem $6.5, \mathfrak{r}=z_{K}$ is the $\ll_{K}^{\triangleright}$-radical and $\rho=\mathbf{1}-\mathfrak{r}$ is the dual $\ll_{K}^{\triangleright}$-radical.

(ii) By Corollary 6.4, $z \rightarrow \ll_{[\mathbf{0}, z]}$ is an injective map from $Q \cap \mathfrak{Z}$ to the set of all RR-orders. If $\ll$ is an $\mathbf{R R}$-order then, by Theorem $6.5, \ll=\ll_{[\mathbf{0}, \mathfrak{r}]}$, where $\mathfrak{r} \in Q \cap \mathfrak{Z}$. The proof is complete.

\section{HH-relation in the lattices of projections in factors.}

In this section $M$ is a factor, i.e., $\mathfrak{Z}=\mathbb{C} 1$, and $Q=P(M)$. So Corollary 6.6 yields

C3.5 Corollary 7.1 Let $M$ be a factor.

(i) There are only two $\mathbf{R R}$-orders : $\ll_{\{\mathbf{0}\}}$ that coincides with $=$ and $\ll_{Q}=\leq$.

(ii) For each trail $K \neq\{\mathbf{0}\}, z_{K}=\vee K=\mathbf{1}$ is the $\ll_{K}^{\triangleright}$-radical and $\mathbf{0}$ is the dual $\ll_{K}^{\triangleright}$-radical.

As $M$ is a factor, then (see [Sa, Corollary 2.1.7])

$$
\text { either } p \prec q \text {, or } p \sim q \text {, or } q \prec p \text { for all } p, q \in Q=P(M) \text {. }
$$

Hence the set $\Omega$ of all orbits in $Q$ is totally ordered and the set $\mathcal{K}_{M}$ of all trails in $Q$ is given in (3.6): $K_{\Phi}=\cup_{\omega \in \Phi} K_{\omega}$, where $\Phi$ is a subset of $\Omega$ and $K_{\omega}=\cup\left\{\omega^{\prime}: \omega^{\prime} \precsim \omega\right\}$ (see (3.5)). 
Let $M$ be a finite factor. By Theorems 8.4.3 and 8.4.4 [KR], there is a unique faithful tracial weight $\tau: Q \rightarrow \mathbb{R}_{+} \cup\{0\}$ such that

$$
\text { 1) } \tau(p \oplus q)=\tau(p)+\tau(q) \text {; 2) } p \precsim q \text { if and only if } \tau(p) \leq \tau(q) \text {. }
$$

If $M$ is a type $\mathrm{I}_{n}$ factor, $\tau$ generates an isomorphism of $\Omega$ onto the set $\{0,1, \ldots, n\}$. So $\Omega=\left\{\omega_{k}\right\}_{k=0}^{n}$, where $\omega_{k}=\{p \in Q: \operatorname{dim} p=\tau(p)=k\}, \omega_{0}=\{\mathbf{0}\}, \omega_{n}=\{\mathbf{1}\}$. Then $\omega_{k} \precsim \omega_{m}$ if $k \leq m$, and

$$
\mathcal{K}_{M}=\left\{K_{\omega_{k}}\right\}_{k=0}^{n}, \text { where } K_{\omega_{k}}=\cup_{i=0}^{k} \omega_{i}=\{p \in Q: \tau(p) \leq k\}, K_{\omega_{0}}=\{\mathbf{0}\} \text { and } K_{\omega_{n}}=Q .
$$

The corresponding HH-relations have form (see (4.1))

$$
p \ll_{K_{\omega_{k}}} q \text { if } p \leq q \text { and } q-p \in K_{\omega_{k}} \text {, i.e., } \tau(q-p) \leq k .
$$

If $M$ is a type $\mathrm{II}_{1}$ factor, $\tau$ generates an isomorphism of $\Omega$ onto $[0,1] \subset \mathbb{R}$. So $\Omega=\left\{\omega_{t}\right\}_{t \in[0,1]}$, where $\omega_{t}=\{p \in Q: \tau(p)=t\}, \omega_{0}=\{\mathbf{0}\}$ and $\omega_{1}=\{\mathbf{1}\}$. Then $\omega_{t} \precsim \omega_{s}$ if $t \leq s$, and

$$
\begin{aligned}
& \mathcal{K}_{M}=\left\{K_{\omega_{t}}\right\}_{t \in[0,1]} \cup\left\{K_{\omega_{t}}^{-}\right\}_{t \in(0,1]}, \text { where } \\
& K_{\omega_{t}}=\cup_{s \in[0, t]} \omega_{s}=\{p \in Q: \tau(p) \leq t\} \text { and } K_{\omega_{t}}^{-}=K_{\omega_{t}} \backslash \omega_{t}=\{p \in Q: \tau(p)<t\} .
\end{aligned}
$$

For $p \leq q$, the corresponding $\mathbf{H H}$-relations have form (see (4.1))

$$
p \ll_{K_{\omega_{t}}} q \text { if } \tau(q-p) \leq t \text {; and } p \ll_{K_{\bar{\omega}_{t}}} q \text { if } \tau(q-p)<t .
$$

T4.1 Theorem 7.2 (i) If $M$ is a type $\mathrm{I}_{n}$ factor then all $\mathbf{H H}$-relations in $Q$ have form given in (7.3). Only two of them are transitive: $\ll_{K_{\omega_{n}}}=\ll_{Q}=\leq$ and $\ll_{K_{\omega_{0}}}=\ll_{\{\mathbf{0}\}}$ coincides with $=$.

(ii) If $M$ is a type $\mathrm{II}_{1}$ factor then all $\mathbf{H H}$-relations in $Q$ have form given in (7.4)). Only two of them are transitive: $\ll_{K_{\omega_{1}}}=\ll_{Q}=\leq$ and $\ll_{K_{\omega_{0}}}=\ll_{\{0\}}$ coincides with $=$.

Proof. (i) follows from the fact that, if $M$ is finite then the map $\ll \rightarrow K^{\ll}$ is an isomorphism of the set of all HH-relations in $Q$ onto $\mathcal{K}_{M}$ by Corollary 4.8. As $S^{{ }_{K} \omega_{k}}=\{p \in Q: \operatorname{dim} p \leq k\}$ is a lattice only if $k=0$ or $n$, we have from Theorem 6.1 that $\ll_{K_{\omega_{k}}}$ is transitive only if $k=0$ or $n$.

(ii) As in (i), we need to prove that only $\ll_{K \omega_{1}}$ and $\ll_{K \omega_{0}}$ are transitive. By (7.2),

$$
\tau(p \oplus q)=\tau(p)+\tau(q) \text { for } p, q \in Q, p \perp q, \text { and } \tau(\mathbf{1})=1 .
$$

Let $t \neq 0$. Then $K_{\omega_{t}}=\{p \in Q: \tau(p) \leq t\}$. Let $K_{\omega_{t}}$ be a lattice.

If $t \leq \frac{1}{2}$, choose $p \in K_{\omega_{t}}$ with $\tau(p)=t$. Then $\tau\left(p^{\perp}\right) \geq \frac{1}{2}$ by (7.5). Hence, by (7.2) and (7.1), $p \precsim p^{\perp}$. By (3.1), there is $r \leq p^{\perp}$ such that $p \sim r$. Hence $\tau(r)=t$. So $r \in K_{\omega_{t}}$. As $K_{\omega_{t}}$ is a lattice, $p \oplus r \in K_{\omega_{t}}$ by (6.1), and $\tau(p \oplus r)=\tau(p)+\tau(r)=2 t>t$ by (7.5), a contradiction. Thus $K_{\omega_{t}}$ is not a lattice if $0<t \leq \frac{1}{2}$.

If $\frac{1}{2}<t<1$, choose $p \in K_{\omega_{t}}$ with $\tau(p)=\frac{1}{2}$. By (7.5), $\tau\left(p^{\perp}\right)=\frac{1}{2}$, so that $p^{\perp} \in K_{\omega_{t}}$. As $K_{\omega_{t}}$ is a lattice, $\mathbf{1}=p \oplus p^{\perp} \in K_{\omega_{t}}$, a contradiction, since $\tau(\mathbf{1})=1>t$. Hence $K_{\omega_{t}}$ is not a lattice. So only $K_{\omega_{0}}=\{\boldsymbol{0}\}$ and $K_{\omega_{1}}=Q$ are lattices. Similarly, all trails $K_{\omega_{t}}^{-}=\{p \in Q: \tau(p)<t\}, 0<t \leq 1$, are not lattices. Thus, by Theorem 6.1, only $\ll_{K_{\omega_{1}}}$ and $\ll_{K_{\omega_{0}}}$ are transitive. 
We will now describe $\mathbf{H H}$-relations in $Q=P(M)$ when $M$ is a type $\mathrm{I}_{\infty}$, or $\mathrm{II}_{\infty}$ factor. By Propositions 8.5.2 and 8.5.5 [KR] (see also Theorems VII.36.1 and VII.36.2 [Na]), there is a faithful normal semi-finite tracial weight $\tau: M^{+} \rightarrow[0, \infty]$ such that, for $p, q \in Q$,

$$
\begin{aligned}
& \text { 1) } \tau(p)=\tau(q) \text { if } p \sim q \text {; } 2) \tau(p \oplus q)=\tau(p)+\tau(q) \text { if } p \perp q \text {; } \\
& \text { 3) } \tau(p)<\tau(q) \text { if } p \prec q \text { and } p \text { is finite; 4) } p \in Q \text { is finite if and only if } \tau(p)<\infty \text {. }
\end{aligned}
$$

Thus $\tau$ is well defined on the totally ordered set of orbits $\Omega$ in $Q: \tau(\omega)=\tau(p), p \in \omega$. Set

$$
\Omega_{\mathrm{inf}}=\{\omega \in \Omega: \tau(\omega)=\infty\} \text { and } \omega_{\sim 1}=\{p \in P(M): p \sim \mathbf{1}\} \in \Omega_{\mathrm{inf}} .
$$

If $M$ is a type $\mathrm{I}_{\infty}$ factor then $\operatorname{range}(\tau)=\mathbb{N} \cup \infty$. Thus (see (3.6))

$$
\Omega=\left\{\omega_{k}\right\}_{k \in \mathbb{N}} \cup \Omega_{\text {inf }}, \text { where } \omega_{k}=\{p \in Q: \operatorname{dim} p=\tau(p)=k\}, \omega_{k} \precsim \omega_{m} \text { if } k \leq m,
$$

and $\omega_{k} \prec \omega$ for all $\omega \in \Omega_{\text {inf }}$. This allows us to describe all trails in $Q$ :

$$
\begin{aligned}
\mathcal{K}_{M} & =\left\{K_{\omega_{k}}\right\}_{k \in \mathbb{N}} \cup K_{\Phi_{\mathbb{N}}} \cup\left\{K_{\Phi}\right\}_{\Phi \subseteq \Omega_{\mathrm{inf}}}, \text { where } \Phi_{\mathbb{N}}=\left\{\omega_{i}\right\}_{i \in \mathbb{N}}, \\
K_{\omega_{k}} & =\cup_{i=0}^{k} \omega_{i}=\{p \in Q: \tau(p) \leq k\}, K_{\Phi_{\mathbb{N}}}=\cup_{i \in \mathbb{N}} \omega_{i}=\{p \in Q: \tau(p)<\infty\}, \\
K_{\omega} & =\cup\left\{\omega^{\prime}: \omega^{\prime} \precsim \omega\right\} \text { and } K_{\Phi}=\cup_{\omega \in \Phi} K_{\omega} \text { for any } \Phi \subseteq \Omega_{\text {inf }} .
\end{aligned}
$$

If $M$ is a type $\mathrm{II}_{\infty}$ factor then $\operatorname{range}(\tau)=[0, \infty]$. Thus (see $\left.(3.6)\right)$

$$
\Omega=\left\{\omega_{t}\right\}_{t \in[0, \infty)} \cup \Omega_{\text {inf }}, \text { where } \omega_{t}=\{p \in Q: \tau(p)=t\}, \omega_{s} \precsim \omega_{t} \text { if } s \leq t,
$$

and $\omega_{t} \prec \omega$ for all $\omega \in \Omega_{\text {inf }}$. This allows us to describe all trails in $Q$ :

$$
\begin{aligned}
& \mathcal{K}_{M}=\left\{K_{\omega_{t}}\right\}_{t \in[0, \infty)} \cup\left\{K_{\omega_{t}}^{-}\right\}_{t \in(0, \infty)} \cup K_{\Phi_{\mathbb{R}}} \cup\left\{K_{\Phi}\right\}_{\Phi \subseteq \Omega_{\mathrm{inf}}}, \text { where } \Phi_{\mathbb{R}}=\left\{\omega_{t}\right\}_{t \in[0, \infty)}, \\
& K_{\omega_{t}}=\cup\left\{\omega_{s}: s \leq t\right\}=\{p \in Q: \tau(p) \leq t\}, K_{\omega_{t}}^{-}=\cup\left\{\omega_{s}: s<t\right\}=\{p \in Q: \tau(p)<t\}, \\
& K_{\Phi_{\mathbb{R}}}=\cup\left\{\omega_{s}: 0 \leq s<\infty\right\}=\{p \in Q: \tau(p)<\infty\} \text { and } K_{\Phi}=\cup_{\omega \in \Phi} K_{\omega} \text { for } \Phi \subseteq \Omega_{\text {inf }} .
\end{aligned}
$$

Recall that, for each $K \in \mathcal{K}_{M} \backslash Q$, the relation $\ll_{\perp}^{K}$ associated with $K$ was defined in (5.6).

T4.2 Theorem 7.3 Let $M$ be a type $\mathrm{I}_{\infty}$ (resp., type $\left.\mathrm{II}_{\infty}\right)$ factor. All relations $\left\{\ll_{K}\right\}_{K \in \mathcal{K}_{M}}$ and $\left\{\ll_{\perp}^{K}\right.$ \}$_{K \in \mathcal{K}_{M} \backslash Q}$ are $\mathbf{H H}$-relations in $Q$. All relations $\ll_{\perp}^{K}$ are not generated by trails.

Only the relations $\ll_{K_{\omega_{0}}}, \ll_{K_{\Phi_{\mathbb{N}}}}\left(\right.$ resp. $\left.\ll_{K_{\Phi_{\mathbb{R}}}}\right)$ and $\ll_{K_{\Phi}}$ for all $\Phi \subseteq \Omega_{\mathrm{inf}}$, are transitive.

Proof. All the results of the theorem follow from (4.14) and Theorem 5.3 apart from transitivity.

Since the relation $\ll_{K_{\omega_{0}}}$ coincides with $=$, it is transitive. As relations $\left\{\ll_{\perp}^{K}\right\}_{K \in \mathcal{K}_{M} \backslash Q}$ are not generated by trails, it follows from Theorem 6.1 that they are not transitive.

Let $M=B(H)$ and $\operatorname{dim} H=\infty$. By (7.8), $K_{\omega_{k}}=\{p \in Q: \operatorname{dim} p \leq k\}$. As it is not a lattice if $k>0, \ll_{K_{\omega_{k}}}$ is not transitive by Theorem 6.1 if $k>0$.

By $(7.8), K_{\omega_{\mathbb{N}}}=\{p \in Q: \operatorname{dim} p<\infty\}$. As it is a lattice, $\ll_{K_{\omega_{\mathbb{N}}}}$ is transitive by Theorem 6.1.

Let $\Phi$ be a subset of $\Omega_{\text {inf }}$ and let $s, t \in K_{\Phi}$ and $s \perp t$. As $M$ is a factor, we may assume, for example, that $s \precsim t$ (see (7.1)). So $s \sim a \leq t$. As $s \in K_{\Phi}$, it follows from (7.6) that it is properly infinite. Hence, by Lemma 6.3.3 [KR], there is $e<s$ such that $e \sim s \ominus e \sim s$. Thus $e \sim a$ and 
$s \ominus e \sim s$. As $e \perp(s \ominus e)$ and $a \perp s$, we have from (3.2) that $a \oplus s \sim e \oplus(s \ominus e)=s \sim a$. Therefore it follows from (3.2) that

$$
t \oplus s=(t \ominus a) \oplus(a \oplus s) \sim(t \ominus a) \oplus a=t \in K_{\Phi} .
$$

Hence it follows from Theorem 6.1 that the relation $\ll_{K_{\Phi}}$ is transitive.

Let $M$ be a $\mathrm{II}_{\infty}$ factor. By (7.9), $K_{\omega_{t}}=\{p \in Q: \tau(p) \leq t\}, t<\infty$. Choose $p \in K_{\omega_{t}}$ such that $\tau(p)=t$. Then $p$ is finite by (7.6), while $p^{\perp}$ is infinite. Hence $p \precsim p^{\perp}$ by (7.1). By (3.1), there is $r \leq p^{\perp}$ such that $p \sim r$. Hence $\tau(r)=t$, so that $r \in K_{\omega_{t}}$ and $p \perp r$. If $K_{\omega_{t}}$ is a lattice, $p \oplus r \in K_{\omega_{t}}$ and $\tau(p \oplus r)=\tau(p)+\tau(r)=2 t$ by (7.6), a contradiction. Thus $K_{\omega_{t}}$ is not a lattice. So $\ll_{K_{\omega t}}$ is not transitive by Theorem 6.1. Similarly, all relations $\ll_{K_{\omega_{t}}}, t<\infty$, are not transitive.

By $(7.9), K_{\omega_{\mathbb{R}}}=\{p \in Q: \tau(p)<\infty\}$, i.e., $K_{\omega_{\mathbb{R}}}=F$ is the set of all finite projections in $Q$. As it is a lattice, $\ll_{K_{\omega_{\mathbb{R}}}}$ is transitive by Theorem 6.1. Repeating the argument used for $M=B(H)$, we get that $\ll_{K_{\Phi}}$ are transitive relations for all $\Phi \subseteq \Omega_{\text {inf }}$ if $M$ is a type $\mathrm{II}_{\infty}$ factor.

For type $\mathrm{I}_{\infty}$ and $\mathrm{II}_{\infty}$ factors $M$, Theorem 7.3 gives a large variety of $\mathbf{H H}$-relations in $Q=P(M)$. Some of them are generated by trails and some are associated with trails, but not generated by them. The question arises as to whether there are other $\mathbf{H H}$-relations in $Q$ which are neither generated by trails, nor associated with them. In Theorem 7.7 we will show that no other HH-relations in $Q$ exist, if $M$ is countably decomposable.

Recall that $M$ is countably decomposable, if each family of mutually orthogonal projections in $M$ is countable (for example, $M \subseteq B(H)$ and $H$ is separable). By Proposition V.1.39 [T], in this case

all infinite projections in $Q$ are equivalent: $\Omega_{\text {inf }}=\left\{\omega_{\sim 1}\right\}=\{p \in P(M): \tau(p)=\infty\}$.

As before, we denote by $F$ the set of all finite projections in $Q=P(M)$.

L4.1 Lemma 7.4 Let $M$ be a not finite factor and $\ll$ be an $\mathbf{H H}$-relation in $Q$. If $\ll$ is not generated by a trail then $F \varsubsetneqq S^{\ll}$. If $M$ is countably decomposable then $K^{\ll}=Q$.

Proof. By Corollary 5.1, $S^{\ll}$ contains an infinite projection $s$. By $(4.11),[\mathbf{0}, s] \subseteq S^{\ll}$. If $p \in F$ then $p \prec s$ by (7.1). Hence there is a finite projection $r<s$ such that $p \sim r$. Then $r \in S^{\ll} \cap F$. As $S^{\ll} \cap F$ is a trail by Corollary 4.7(i), $p \in S^{\ll}$. Thus $F \varsubsetneqq S^{\ll}$.

If $M$ is countably decomposable, $s \sim \mathbf{1}$ by (7.10). Thus $\mathbf{1} \in K^{\ll}$, so that $K^{\ll}=Q$ by (4.11).

We need now the following general result.

L3.1 Lemma 7.5 Let $M$ be a factor and $p \wedge q=\mathbf{0}$. Set $a=p \wedge q^{\perp}, c=p^{\perp} \wedge q, e=p^{\perp} \wedge q^{\perp}$.

(i) There is $d \sim p$ such that $q^{\perp}=d \oplus e$.

(ii) There is $r \in Q$ such that $r \wedge p=r \wedge q=\mathbf{0}$ and either $r \sim q^{\perp}$ if $a \precsim c$, or $r \sim p^{\perp}$ if $c \precsim a$.

Proof. As $p \wedge q=\mathbf{0}$, it follows from (4.12) that there are projections $p_{0}, q_{0}$ such that

$$
\begin{aligned}
& p=p_{0} \oplus a, q=q_{0} \oplus c, q^{\perp}=\left(b \ominus q_{0}\right) \oplus a \oplus e, \mathbf{1}=b \oplus a \oplus c \oplus e, \text { where } \\
& b=p_{0} \vee q_{0}, p_{0} \sim b \ominus q_{0} \sim q_{0} \sim b \ominus p_{0} \text { and } \\
& \mathbf{0}=p_{0} \wedge q_{0}=p_{0} \wedge\left(b \ominus q_{0}\right)=\left(b \ominus p_{0}\right) \wedge q_{0} .
\end{aligned}
$$


(i) Set $d=\left(b \ominus q_{0}\right) \oplus a$. By (3.2), (7.11) and (7.12), $p \sim d$ and $q^{\perp}=d \oplus e$.

(ii) As $M$ is a factor, either $a \precsim c$, or $c \precsim a$. Let $a \precsim c$. Then there is $s \leq c$ such that $a \sim s$. As $c \perp a$, we have $s \perp a$. Hence, by Lemma 4.4(ii), there is $t \in Q$ such that

$$
t \leq s \oplus a \leq c \oplus a, t \wedge a=t \wedge s=\mathbf{0}, t \sim a \sim s \text { and } t \oplus a=s \oplus a .
$$

Set $r=\left(b \ominus q_{0}\right) \oplus t \oplus e$. By (7.11) and (7.14), $p_{0}, q_{0} \perp(t \oplus e)$ and $(b \oplus e) \perp(a \oplus c)$. So

$$
\begin{aligned}
r \wedge p & =\left(\left(b \ominus q_{0}\right) \oplus t \oplus e\right) \wedge\left(p_{0} \oplus a\right) \stackrel{(4.7)}{=}\left(\left(b \ominus q_{0}\right) \wedge p_{0}\right) \oplus(t \wedge a) \stackrel{(7.13)}{=} \mathbf{0} \oplus(t \wedge a) \stackrel{(7.14)}{=} \mathbf{0}, \\
r \wedge q & =\left(\left(b \ominus q_{0}\right) \oplus t \oplus e\right) \wedge\left(q_{0} \oplus c\right) \stackrel{(4.7)}{=}\left(\left(b \ominus q_{0}\right) \wedge q_{0}\right) \oplus(t \wedge c) \stackrel{(7.13)}{=} \mathbf{0} \oplus(t \wedge c) \\
& =t \wedge(s \oplus(c \ominus s)) \stackrel{(4.7)}{=} t \wedge s \stackrel{(7.14)}{=} \mathbf{0},
\end{aligned}
$$

since $t \perp(c \ominus s)$. As $t \sim a$ by (7.14), it follows from (7.11) that $r \sim q^{\perp}$.

Similarly, one can prove (ii) if $c \precsim a$.

We shall now study the sets $S^{\ll}$ for $\mathbf{H H}$-relations $\ll$ when $M$ are $\mathrm{I}_{\infty}$ and $\mathrm{II}_{\infty}$ factors.

P4.3 Proposition 7.6 Let $M$ be a factor, $\ll$ be an $\mathbf{H H}$-relation and $\tau: M^{+} \rightarrow[0, \infty]$ satisfy (7.6). If

$$
\tau\left(p^{\perp}\right)=\alpha<\infty \text { for some } p \in S^{\ll} \text {, then }\left\{q \in Q: \alpha \leq \tau\left(q^{\perp}\right)\right\} \subseteq S^{\ll} \text {. }
$$

Proof. If $\alpha=0$ then $p^{\perp}=\mathbf{0}$, so that $p=\mathbf{1}$ and $S^{\ll}=Q$. Let now $\alpha>0$ and $\tau\left(q^{\perp}\right)=\alpha$. By (7.6), $p^{\perp} \sim q^{\perp}$. To prove that $q \in S^{\ll}$, we first show that there is $r \in Q$ such that

$$
\tau(r)=\alpha, r \wedge p=r \wedge q=\mathbf{0} \text { and } r \vee p=\mathbf{1} .
$$

Set $s=p \wedge q, p_{1}=p s^{\perp}=p-p s, q_{1}=q s^{\perp}=q-q s$. Then $s^{\perp}-q_{1}=s^{\perp} q^{\perp}=q^{\perp}$, as $q^{\perp} \leq s^{\perp}$. So

$$
p_{1} \wedge q_{1}=p s^{\perp} \wedge q s^{\perp} \stackrel{(4.5)}{=}(p \wedge q) s^{\perp}=s s^{\perp}=\mathbf{0} \text {, as } s^{\perp} \text { commutes with } p \text { and } q .
$$

Consider the factor $M_{s^{\perp}}=s^{\perp} M s^{\perp}=\left\{s^{\perp} a s^{\perp}: a \in M\right\}$. Then $p_{1}, q_{1} \in M_{s^{\perp}}$. As $p_{1} \wedge q_{1}=\mathbf{0}$, it follows from Lemma 7.5(ii) that there is $r \in P\left(M_{s^{\perp}}\right)$ such that $r \wedge p_{1}=r \wedge q_{1}=\mathbf{0}$ and $r \sim s^{\perp} \ominus q_{1}=q^{\perp} \sim p^{\perp}$.

As $r \leq s^{\perp}$ and $p=p_{1} \oplus p s$, we have $r \perp p s$ and $r \wedge p=r \wedge\left(p_{1} \oplus p s\right) \stackrel{(4.7)}{=} r \wedge p_{1}=\mathbf{0}$. Similarly, $r \wedge q=\mathbf{0}$. Since $r \sim q^{\perp}$, we have $\tau(r)=\tau\left(q^{\perp}\right)=\alpha$ by (7.6).

To prove that $r \vee p=\mathbf{1}$, recall that $r \wedge p=\mathbf{0}$. Replacing $q$ by $r$ in (7.11)-(7.13), we get

$$
r=r_{0} \oplus\left(r \wedge p^{\perp}\right), p^{\perp}=\left(b \ominus p_{0}\right) \oplus\left(r \wedge p^{\perp}\right) \oplus\left(r^{\perp} \wedge p^{\perp}\right), b=p_{0} \vee r_{0} \text { and } r_{0} \sim b \ominus p_{0},
$$

where $b=r_{0} \vee p_{0}$. As $\tau\left(p^{\perp}\right)=\alpha=\tau(r)$, it follows from (7.17) and (7.6) that

$$
\alpha=\tau(r)=\tau\left(r_{0}\right)+\tau\left(r \wedge p^{\perp}\right) \text { and } \alpha=\tau\left(p^{\perp}\right)=\tau\left(b \ominus p_{0}\right)+\tau\left(r \wedge p^{\perp}\right)+\tau\left(r^{\perp} \wedge p^{\perp}\right) .
$$

As $r_{0} \sim b \ominus p_{0}$ by (7.17), we have $\tau\left(r_{0}\right)=\tau\left(b \ominus p_{0}\right)$ by (7.6). Hence $\tau\left(r^{\perp} \wedge p^{\perp}\right)=0$. As $\tau$ is faithful, $r^{\perp} \wedge p^{\perp}=\mathbf{0}$. So $r \vee p=\left(r^{\perp} \wedge p^{\perp}\right)^{\perp}=\mathbf{1}$ by (4.4). Thus (7.16) is proved.

By (7.16) and Lemma 4.4(i),

$$
p \in S^{\ll} \text { and } \tau\left(p^{\perp}\right)=\tau\left(q^{\perp}\right)=\alpha \text { imply } \mathbf{0} \ll q \text {, i.e., } q \in S^{\ll} \text {. }
$$

Finally, let $\tau\left(q^{\perp}\right)>\alpha=\tau\left(p^{\perp}\right)$. Then $p^{\perp} \prec q^{\perp}$ by (7.6). So $p^{\perp} \sim t<q^{\perp}$ for some $t \in Q$. Thus $\tau(t)=\alpha$ by (7.6). As $t=\left(t^{\perp}\right)^{\perp}$, we get $t^{\perp} \in S^{\ll}$ from (7.18). By (4.11), $q \in S^{\ll}$, as $q<t^{\perp}$.

Using Proposition 7.6, we will now show that, for a countably decomposable type $\mathrm{I}_{\infty}$, or $\mathrm{II}_{\infty}$ factor $M$, Theorem 7.3 gives a full list of $\mathbf{H H}$-relations in $P(M)$. 
T6.3 Theorem 7.7 Let $M$ be a type $\mathrm{I}_{\infty}$, or $\mathrm{II}_{\infty}$ factor. Let $\ll$ be an $\mathbf{H H}$-relation in $Q=P(M)$ not generated by a trail. Set

$$
\alpha=\inf \left\{\tau\left(q^{\perp}\right): q \in S^{\ll}\right\} \text { and let } \omega_{\alpha}=\{p \in Q: \tau(p)=\alpha\} \in \Omega, \text { if } \alpha<\infty .
$$

(i) If $M$ is a type $\mathrm{I}_{\infty}$ factor and $\alpha<\infty$ then $\ll=\ll_{\perp}^{K_{\omega_{\alpha-1}}}$.

(ii) Let $M$ be a type $\mathrm{II}_{\infty}$ factor and $\alpha<\infty$.

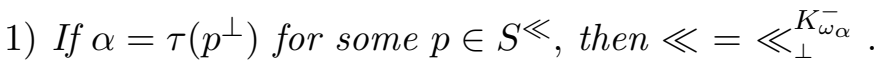

2) If $\alpha<\tau\left(p^{\perp}\right)$ for all $p \in S^{\ll}$, then $\ll=\ll_{\perp}^{K_{\omega_{\alpha}}}$.

(iii) Let $\alpha=\infty$ and $M$ be countably decomposable. If $M$ has type $\mathrm{I}_{\infty}$ then $\ll=\ll_{\perp}^{K_{\Phi_{\mathbb{N}}}}$; if $M$ has type $\mathrm{II}_{\infty}$ then $\ll=\ll_{\perp}^{K_{\Phi_{\mathbb{R}}}}$.

Proof. (i) If $M$ is a type $\mathrm{I}_{\infty}$ factor then $\alpha \in \mathbb{N}$. So there is $p \in S^{\ll}$ such that $\tau\left(p^{\perp}\right)=\alpha$. Then

$$
S^{\ll} \stackrel{K_{\perp} \omega_{\alpha-1}}{\stackrel{(5.7)}{=}}\left\{s \in Q: s^{\perp} \notin K_{\omega_{\alpha-1}}\right\} \stackrel{(7.8)}{=}\left\{s \in Q: \alpha \leq \tau\left(s^{\perp}\right)\right\} \stackrel{(7.15)}{\subseteq} S^{\ll} .
$$

On the other hand, since $\alpha \leq \tau\left(q^{\perp}\right)$ for all $q \in S^{\ll}$ by (7.19), we have $S^{\ll} \subseteq S^{\ll_{\perp}^{K_{\alpha}-1}}$. Thus $S^{\ll}=S^{\ll_{\perp}^{K \omega_{\alpha-1}}}$. Hence, by Lemma $4.3, \ll=\ll_{\perp}^{K \omega_{\alpha-1}}$.

(ii) Let $M$ be a type $\mathrm{II}_{\infty}$ factor. Then $\alpha \in \mathbb{R}_{+} \cup\{0\}$.

1) Let $\alpha=\tau\left(p^{\perp}\right)$ for some $p \in S^{\ll}$. Then

$$
S^{\ll}{ }_{\perp}^{K_{\omega_{\alpha}}} \stackrel{(5.7)}{=}\left\{s \in Q: s^{\perp} \notin K_{\omega_{\alpha}}^{-}\right\} \stackrel{(7.9)}{=}\left\{s \in Q: \alpha \leq \tau\left(s^{\perp}\right)\right\} \stackrel{(7.15)}{\subseteq} S^{\ll} .
$$

On the other hand, since $\alpha \leq \tau\left(q^{\perp}\right)$ for all $q \in S^{\ll}$ by (7.19), we have $S^{\ll} \subseteq S^{\ll_{\perp} \bar{\omega}_{\alpha}}$. Thus $S^{\ll}=S^{\ll_{\perp}^{K_{\omega_{\alpha}}}}$. Hence, by Lemma $4.3, \ll=\ll_{\perp}^{K_{\omega_{\alpha}}^{-}}$.

2) Let $\alpha<\tau\left(p^{\perp}\right)$ for all $p \in S^{\ll}$. For each $\varepsilon>0$, there is $p_{\varepsilon} \in S^{\ll}$ such that $\alpha<\tau\left(p_{\varepsilon}^{\perp}\right) \leq \alpha+\varepsilon$. By (7.15), $\left\{s \in Q: \alpha+\varepsilon \leq \tau\left(s^{\perp}\right)\right\} \subseteq S^{\ll}$. Thus $\left\{s \in Q: \alpha<\tau\left(s^{\perp}\right)\right\} \subseteq S^{\ll}$. As

$$
S^{\ll} \ll_{\perp}^{K_{\alpha}} \stackrel{(5.7)}{=}\left\{s \in Q: s^{\perp} \notin K_{\omega_{\alpha}}\right\} \stackrel{(7.9)}{=}\left\{s \in Q: \alpha<\tau\left(s^{\perp}\right)\right\},
$$

we have $S^{\ll_{\perp}^{K \omega_{\alpha}}} \subseteq S^{\ll}$. On the other hand, as $\tau\left(p^{\perp}\right)>\alpha$ for all $p \in S^{\ll}$, we have $S^{\ll} \subseteq S^{\ll_{\perp}^{K_{\omega_{\alpha}}}}$ by (7.20). Thus $S^{\ll}=S^{\ll}{ }_{\perp}^{K \omega_{\alpha}}$. By Lemma $4.3, \ll=\ll_{\perp}^{K \omega_{\alpha}}$.

(iii) Let $\alpha=\infty$ and $M$ be countably decomposable. Then $\Omega_{\text {inf }}=\left\{\omega_{\sim 1}\right\}$ (see (7.10)). As

$$
\tau\left(p^{\perp}\right)=\infty \text { for all } p \in S^{\ll} \text { by (7.19), we have } p^{\perp} \in \omega_{\sim 1} .
$$

Let $M$ have type $\mathrm{I}_{\infty}$. By $(7.5), K:=K_{\Phi_{\mathbb{N}}}=\cup_{i \in \mathbb{N}} \omega_{i}=\{p \in Q: \tau(p)<\infty\}$. Then

$$
\begin{aligned}
& S_{\perp}^{\ll} \stackrel{(5.7)}{=}\left\{q \in Q: q^{\perp} \notin K\right\} \stackrel{(7.8)}{=}\left\{q \in Q: \tau\left(q^{\perp}\right)=\infty\right\}=\left\{q \in Q: q^{\perp} \in \omega_{\sim 1}\right\}, \\
& \text { so that } S^{\ll} \subseteq S^{\ll_{\perp}^{K}} \text { by }(7.21) .
\end{aligned}
$$

By Lemma $7.4, F \subseteq S^{\ll}$ and $S^{\ll}$ contains infinite projections. Then

$$
S^{\ll}=F \cup S_{\infty}^{\ll} \text {, where } S_{\infty}^{\ll}=\left\{p \in S^{\ll:} p \text { is infinite } \stackrel{(7.21)}{=}\left\{p \in S^{\ll:} p, p^{\perp} \text { are infinite }\right\}\right. \text {. }
$$


As $M$ is countably decomposable, $p \sim p^{\perp}$ for $p \in S_{\infty}^{\ll}$ by (7.10). Thus, by Proposition 4.5,

$$
\text { if } p \in S_{\infty}^{\ll} \text { then } \mathbf{0} \ll p^{\perp} \text {, so that } p^{\perp} \in S_{\infty}^{\ll} \text {. }
$$

Let $p \in S_{\infty}^{\ll}, p \leq q$ and $q^{\perp}$ be infinite. Then $q^{\perp} \leq p^{\perp} \in S_{\infty}^{\ll}$ by (7.25). By (4.11), $q^{\perp} \in S^{\ll}$. As $q^{\perp}$ is infinite, $q^{\perp} \in S_{\infty}^{\ll}$ by (7.24). Hence, by $(7.25), q=\left(q^{\perp}\right)^{\perp} \in S_{\infty}^{\ll}$. So

$$
\text { if } p \in S_{\infty}^{\ll}, p \leq q \text { and } q^{\perp} \text { is infinite then } q \in S_{\infty}^{\ll} \text {. }
$$

If $p \in F$ then $\tau(p)<\infty$, so that $\tau\left(p^{\perp}\right)=\infty$ by (7.6). So it follows from (7.22) that

$$
S^{\ll_{\perp}^{K}}=F \cup S_{\infty}^{\ll_{\perp}^{K}} \text {, where } S_{\infty}^{\ll_{\perp}^{K}}=\left\{q \in Q: q \text { and } q^{\perp} \text { are infinite }\right\} .
$$

Comparing (7.24) and (7.27), we have that in order to prove

$$
S^{\ll}{ }_{\perp}^{K} \subseteq S^{\ll} \text {, we need to show that } S_{\infty}^{\ll_{\perp}^{K}} \subseteq S_{\infty}^{\ll} \text {. }
$$

Let $q \in S_{\infty}^{\ll}$. Take any $p \in S_{\infty}^{\ll}$. If $(p \vee q)^{\perp}$ is infinite then $p \vee q \in S_{\infty}^{\ll}$ by (7.26), as $p \leq p \vee q$. As $q \leq p \vee q$, we have $q \in S^{\ll}$ by (4.11). As $q$ is infinite (see (7.27)), $q \in S_{\infty}^{\ll}$ by (7.24).

Let now $(p \vee q)^{\perp}$ be finite. As $\mathbf{0} \ll p$, we have $q=\mathbf{0} \vee q \ll p \vee q$ by $(2.1)$, so that $\mathbf{0}=q \wedge q^{\perp} \ll$ $(p \vee q) \wedge q^{\perp}$ by (2.2). Set $r=(p \vee q) \wedge q^{\perp}$. Then $r \in S^{\ll}$, as $\mathbf{0} \ll r$. It follows from (4.4) that

$$
r^{\perp}=\left((p \vee q) \wedge q^{\perp}\right)^{\perp}=(p \vee q)^{\perp} \vee\left(q^{\perp}\right)^{\perp}=\left(p^{\perp} \wedge q^{\perp}\right) \vee q=\left(p^{\perp} \wedge q^{\perp}\right) \oplus q,
$$

as $p^{\perp} \wedge q^{\perp}$ is orthogonal to $q$. Hence

$$
r=\left(r^{\perp}\right)^{\perp}=\left(\left(p^{\perp} \wedge q^{\perp}\right) \oplus q\right)^{\perp}=q^{\perp} \ominus\left(p^{\perp} \wedge q^{\perp}\right) .
$$

As $q \in S_{\infty}^{\ll^{K}}, q^{\perp}$ is infinite by (7.27). Hence $r$ is infinite, since $\left(p^{\perp} \wedge q^{\perp}\right)=(p \vee q)^{\perp}$ is finite by our assumption. As $r \in S^{\ll}$, we have $r \in S_{\infty}^{\ll}$ by (7.24). As $q^{\perp}=r \oplus\left(p^{\perp} \wedge q^{\perp}\right) \geq r$ by (7.29), and $\left(q^{\perp}\right)^{\perp}=q$ is infinite, it follows from (7.26) that $q^{\perp} \in S_{\infty}^{\ll}$. Then $q \in S_{\infty}^{\ll}$ by (7.25). Thus $S_{\infty}^{\ll_{\perp}^{K}} \subseteq S_{\infty}^{\ll}$, so that $S^{\ll_{\perp}^{K}} \subseteq S^{\ll}$ by (7.28). Combining this with (7.23) yields $S^{\ll_{\perp}^{K}}=S^{\ll}$. By Lemma $4.3, \ll=\ll_{\perp}^{K}=\ll_{\perp}^{\bar{K}_{\Phi_{N}}}$.

The proof of $\ll=\ll_{\perp}^{K_{\Phi_{\mathbb{R}}}}$ if $M$ has type $\mathrm{II}_{\infty}$, is identical.

To summarize the results of Theorems 7.3 and 7.7, assume that $M$ is a countably decomposable factor. Then $\Omega_{\text {inf }}=\left\{\omega_{\sim 1}\right\}$. Hence if $K \in \mathcal{K}_{M}$ contains an infinite projection then $K=Q$. For each $K \in \mathcal{K}_{M}$, the corresponding $\mathbf{H H}$-relations $\ll_{K}$ and $\ll_{\perp}^{K}$ are given in (4.1) and (5.6).

If $M$ has type $\mathrm{I}_{\infty}$ then (see (7.8)) $\mathcal{K}_{M}=\left\{K_{\omega_{k}}\right\}_{k \in \mathbb{N}} \cup K_{\Phi_{\mathbb{N}}} \cup Q$, where $K_{\omega_{k}}=\{p \in Q: \tau(p) \leq k\}$ and $K_{\Phi_{\mathbb{N}}}=\{p \in Q: \tau(p)<\infty\}$. The corresponding HH-relations have form: for $p \leq q$,

$$
\begin{aligned}
& p \ll_{K_{\omega_{k}}} q \text { if } \tau(q-p) \leq k, \text { and } p \ll_{\perp}^{K_{\omega_{k}}} q \text { if } \tau\left((q-p)^{\perp}\right)>k, \text { for } k \in \mathbb{N} ; \\
& p \ll_{K_{\Phi_{\mathbb{N}}}} q \text { if } \tau(q-p)<\infty \text {, and } p \ll_{\perp}^{K_{\Phi_{\mathbb{N}}}} q \text { if } \tau\left((q-p)^{\perp}\right)=\infty .
\end{aligned}
$$

If $M$ has type $\mathrm{II}_{\infty}$ then (see (7.9)) $\mathcal{K}_{M}=\left\{K_{\omega_{t}}\right\}_{t \in[0, \infty)} \cup\left\{K_{\omega_{t}}^{-}\right\}_{t \in(0, \infty)} \cup K_{\Phi_{\mathbb{R}}} \cup Q$, where $K_{\omega_{t}}=\{p \in Q$ : $\tau(p) \leq t\}, K_{\omega_{t}}^{-}=\{p \in Q: \tau(p)<t\}$ and $K_{\Phi_{\mathbb{R}}}=\{p \in Q: \tau(p)<\infty\}$. The corresponding HHrelations have form: for $p \leq q$, 


$$
\begin{aligned}
& p \ll_{K_{\omega_{t}}} q \text { if } \tau(q-p) \leq t, \text { and } p \ll_{\perp}^{K_{\omega_{t}}} q \text { if } \tau\left((q-p)^{\perp}\right)>t \text { for } t \in[0, \infty), \\
& p \ll_{K_{\bar{\omega}_{t}}} q \text { if } \tau(q-p)<t, \text { and } p \ll_{\perp}^{K_{\omega_{t}}^{-}} q \text { if } \tau\left((q-p)^{\perp}\right) \geq t \text { for } t \in(0, \infty) \\
& p \ll_{K_{\Phi_{\mathbb{R}}}} q \text { if } \tau(q-p)<\infty, \text { and } p \ll_{\perp}^{K_{\Phi_{\mathbb{R}}}} q \text { if } \tau\left((q-p)^{\perp}\right)=\infty
\end{aligned}
$$

In both cases, $\ll_{Q}=\leq$. Therefore Theorems 7.3 and 7.7 yield.

T7.1 Theorem 7.8 Let $M$ be a countably decomposable factor of type $\mathrm{I}_{\infty}$ (resp. $\left.\mathrm{II}_{\infty}\right)$. A relation in $Q$ is an HH-relation if and only if it coincides with one of the relations in (7.30) (resp. in (7.31)). Only three relations are transitive: $\ll_{Q}=\leq, \ll_{K_{\Phi_{\mathbb{N}}}}\left(\right.$ resp., $\left.\ll_{K_{\Phi_{\mathbb{R}}}}\right), \ll_{K_{\omega_{0}}}$ that coincides with $=$.

Consider now HH-relations in $Q=P(M)$, when $M$ is a countably decomposable type III factor. Note that $M$ has no non-trivial faithful semi-finite normal tracial weights (Theorem V.2.15 [T]).

T6.1 Theorem 7.9 Let $M$ be a countably decomposable type III factor. Then $Q$ only has two trails: $K_{\mathbf{0}}=\{\mathbf{0}\}$ and $K_{\mathbf{1}}=Q$ and only three $\mathbf{H H}$-relations. Two of them: $\ll_{K_{\mathbf{1}}}=\leq$ and $\ll_{K_{\mathbf{0}}}$ that coincides with $=$ are generated by trails. Only they are transitive.

The third relation $\ll_{\perp}^{K_{\mathbf{0}}}$ is not generated by a trail: $p \ll_{\perp}^{K_{\mathbf{0}}} q$ if $p \leq q$ and $q-p \neq \mathbf{1}$.

Proof. As all projections in $Q \backslash\{\mathbf{0}\}$ are infinite and equivalent to $\mathbf{1}$ ([T], V.1.39), $Q$ only has two trails $K_{\mathbf{0}}=\{\mathbf{0}\}$ and $K_{\mathbf{1}}=Q$ and two $\mathbf{H H}$-relations generated by them.

Let an HH-relation $\ll$ be not generated by a trail. If $\mathbf{1} \in S^{\ll}$ then $Q=[\mathbf{0}, \mathbf{1}] \subseteq S^{\ll}$ by (4.11), a contradiction. Thus $\mathbf{1} \notin S^{\ll}$. Let $\mathbf{0} \neq p \in S^{\ll}$. Then $p \sim p^{\perp} \neq \mathbf{0}, \mathbf{1}$. Hence, by Proposition 4.5,

$$
\mathbf{0} \ll p \text { implies } p^{\perp} \in S^{\ll} \text {, so that }\left[\mathbf{0}, p^{\perp}\right] \subseteq S^{\ll} \text { by }(4.11) \text {. }
$$

If $p \leq q \neq \mathbf{1}$ then $\mathbf{0} \neq q^{\perp} \leq p^{\perp}$. Hence $\mathbf{0} \neq q^{\perp} \in S^{\ll}$. By (7.32), $q=\left(q^{\perp}\right)^{\perp} \in S^{\ll}$. Thus $\mathbf{0} \neq p \in S^{\ll}$ and $p \leq q \neq \mathbf{1}$ implies $q \in S^{\ll}$. If $p \vee r \neq \mathbf{1}$ for some $r \in Q$, then $p \vee r \in S^{\ll}$, as $p \leq p \vee r$. So $r \in S^{\ll}$ by (4.11), as $r \leq p \vee r$.

Let $p \vee r=\mathbf{1}$ and $\mathbf{1} \neq r$. Then $\mathbf{0}=\mathbf{1}^{\perp}=(p \vee r)^{\perp}=p^{\perp} \wedge r^{\perp}$ by (4.4). As $\mathbf{0} \ll p$, we have $p^{\perp}=\mathbf{0} \vee p^{\perp} \ll p \vee p^{\perp}=\mathbf{1}$ by (2.1). Hence $\mathbf{0}=p^{\perp} \wedge r^{\perp} \ll \mathbf{1} \wedge r^{\perp}=r^{\perp}$. By (7.32), $r=\left(r^{\perp}\right)^{\perp} \in S^{\ll}$. Thus each $r \neq \mathbf{1}$ belongs to $S^{\ll}$. So $S^{\ll}=Q \backslash\{\mathbf{1}\}$. Therefore $\ll=\ll_{Q \backslash\{\mathbf{1}\}}=\ll_{\perp}^{K_{0}}$ (see $\left.(5.6)\right)$.

P1 Problem 7.10 For a countably decomposable infinite factor $M$, the list of $\mathbf{H H}$-relations in $P(M)$ given in Theorem 7.3 is complete by Theorems 7.8 and 7.9. Is it also complete if $M$ is not countably decomposable?

\section{References}

A1 [A1] S. A. Amitsur, A general theory of radicals. I. Radicals in complete lattices, Amer. J. Math. 74 (1952) 774-786.

A2 [A2] S. A. Amitsur, A general theory of radicals. II. Radicals in rings and bicategories; and III. Applications, Amer. J. Math. 76 (1954) 100-125 and 126-136.

Gr [Gr] M. Gray, "A radical approach to algebra", Addison-Wesley, London, Ontario, 1970. 
KR $\quad \mathrm{KR}]$ R.V. Kadison and J.R. Ringrose, Fundamentals of the theory of operator algebras Vol. I and II, Academic Press, Inc., London, 1986.

KST1 [KST1] E. Kissin, V.S. Shulman and Yu. V. Turovskii, On theory of topological radicals, Journal of Mathematical Sciences, 2018, to appear.

KST [KST] E. Kissin, V.S. Shulman and Yu. V. Turovskii, Relations and radicals in lattices of ideals of $\mathrm{C}^{*}$-algebras, 2018, to appear.

$\mathrm{Na}[\mathrm{Na}]$ M. A. Naimark, "Normed algebras", Wolters-Noordhoff Publishing, Groningen, Netherlands, 1972.

Sa $\quad$ Sa] S. Sakai, "C*-algebras and W*-algebras", Springer-Verlag, New-York Heidelberg, 1998.

T [T] M. Takesaki, "Theory of Operator Algebras I", Springer-Verlag, New-York Heidelberg, 2002.

E. Kissin: STORM, London Metropolitan University, 166-220 Holloway Road, London N7 8DB, Great Britain;

e-mail: e.kissin@londonmet.ac.uk

MATHEMATICS SUBJECT CLASSIFICATION: 46L10, 06C99 